\title{
Pharmacologic HIV-1 Nef blockade promotes CD8 T cell-mediated elimination of latently HIV-1-infected cells in vitro
}

\author{
Shariq Mujib, ${ }^{1}$ Aamir Saiyed, ${ }^{2}$ Saleh Fadel, ${ }^{2}$ Ardalan Bozorgzad, ${ }^{2}$ Nasra Aidarus, ${ }^{2}$ \\ Feng Yun Yue, ${ }^{2}$ Erika Benko, ${ }^{3}$ Colin Kovacs, ${ }^{2,3}$ Lori A. Emert-Sedlak, ${ }^{4}$ Thomas E. Smithgall, ${ }^{4}$ \\ and Mario A. Ostrowski ${ }^{1,2,5}$ \\ IInstitute of Medical Science (IMS), Department of Medicine, and 2Department of Immunology, University of Toronto, \\ Toronto, Ontario, Canada. ${ }^{3}$ Maple Leaf Medical Clinic, Toronto, Ontario, Canada. ${ }^{4}$ Department of Microbiology and \\ Molecular Genetics, University of Pittsburgh, Pittsburgh, Pennsylvania, USA. ${ }^{5}$ Keenan Research Centre for Biomedical \\ Science of St. Michael's Hospital, Toronto, Ontario, Canada.
}

\begin{abstract}
Eradication of the HIV-1 latent reservoir represents the current paradigm to developing a cure for AIDS. HIV-1 has evolved multiple mechanisms to evade CD8 T cell responses, including HIV1 Nef-mediated downregulation of MHC-I from the surface of infected cells. Nef transcripts and protein are detectable in samples from aviremic donors, suggesting that Nef expression in latently HIV-1-infected CD4 T cells protects them from immune-mediated clearance. Here, we tested 4 small molecule inhibitors of HIV-1 Nef in an in vitro primary CD4 T cell latency model and measured the ability of autologous ex vivo or HIV-1 peptide-expanded CD8 T cells to recognize and kill latently infected cells as a function of inhibitor treatment. Nef inhibition enhanced cytokine secretion by autologous CD8 T cells against latently HIV-1-infected targets in an IFN- $\gamma$ release assay. Additionally, CD8 T cell-mediated elimination of latently HIV-1-infected cells was significantly enhanced following Nef blockade, measured as a reduction in the frequency of infected cells and Gag protein in cultures following viral outgrowth assays. We demonstrate for the first time to our knowledge that Nef blockade, in combination with HIV-specific CD8 T cell expansion, might be a feasible strategy to target the HIV-1 latent reservoir that should be tested further in vivo.
\end{abstract}

Conflict of interest: The authors have declared that no conflict of interest exists.

Submitted: February 28, 2017 Accepted: July 25, 2017 Published: September 7, 2017

\section{Reference information:} JCI Insight. 2017;2(17):e93684 https://doi.org/10.1172/jici. insight.93684.

\section{Introduction}

HIV-1 persists indefinitely, despite daily adherence to highly active antiretroviral therapy (HAART) by establishing a lifelong reservoir primarily composed of long-lived memory CD4 T cells (1). Eradication of the reservoir represents the current paradigm to the development of a cure for the more than 36 million individuals living with HIV-1 globally (2). Plasma viral loads typically recede below detection limits on suppressive HAART, but cessation of therapy is marked by rapid viral rebound due to the existence of a latent pool of HIV-1-infected cells (3). The latent reservoir is long lived and maintained, despite effective HAART $(1,4,5)$. Recently, ongoing replication at tissue sites such as lymph nodes has also been described, in part due to poor drug bioavailability $(6,7)$. Furthermore, cell-to-cell spread of HIV-1 infection occurs efficiently, despite the presence of antiretrovirals (8). Additionally, rare T cells expressing HIV-1 proteins have been described and imaged in vivo in the PBMC of HIV-infected individuals on suppressive HAART (9). Other studies have also identified rare HIV-1 Gag protein-expressing CD4 T cells ex vivo in PBMC from HIV-1-infected individuals on suppressive HAART (10), and a recent report has also characterized a subset of dual HIV-1 Gag protein-expressing and RNA-encoding CD4 T cells ex vivo in PBMC samples from individuals on long-term HAART (11). Hence, HIV-1 latency may be described as a state of low-level transcriptional activity, whereby some viral products may be constantly generated. Since HAART alone is not a cure for HIV-1 infection, novel strategies must be employed to develop either a functional (sustained remission off of therapy) or sterilizing (complete reservoir eradication) cure.

Multiply spliced RNA (msRNA) from HIV-1 transcripts, encoding for the regulatory proteins Tat, Rev, and Nef, are readily detected in the PBMC of HIV-1-infected individuals on HAART (12). Of particular interest to us is the HIV-1 product Nef, which has been reported to be present in the PBMC (13), as well as 
in plasma extracellular vesicles (14) of individuals on suppressive HAART. Nef is a $27-35 \mathrm{kDa}$ myristoylated protein involved in a myriad of functions and plays a critical role in disease progression (reviewed in ref. 15). Interestingly, rare HIV-1 Gag-expressing T cells that exhibit a Nef-dependent CD4-downregulated phenotype have been visualized in ex vivo PBMC samples from individuals on suppressive HAART (9), suggesting this accessory protein is actively involved in cellular processes in vivo despite antiretroviral therapy. Nef is crucial for the progression to AIDS, as highlighted by SIV models of infection whereby animals infected with Nef-deficient strains either did not progress to or exhibited a delayed onset of AIDS-like disease (16). Individuals infected with strains of HIV-1 bearing attenuated Nef gene variants are associated with slower, nonprogressive infection (17-19). Downregulation of cell-surface MHC-I (20) is one of Nef's most studied functions (reviewed in ref. 21). Reduced surface expression of MHC-I on HIV-1-infected cells effectively prevents CD8 T cell surveillance, allowing infected cells to persist (22, 23). HIV-specific CD8 T cells are vital for controlling HIV-1 infection, as evidenced by elite controllers, rare individuals that naturally maintain high CD4 counts and low to undetectable viral loads in the absence of HAART due to polyfunctional CD8 T cell clones that help maintain a smaller viral reservoir burden (24-26). Furthermore, it has been observed that rapid expansion of HIV-specific CD8 T cells during the acute phase of infection inversely correlates with viral set point (27). Additionally, CD8 T cells exhibit remarkable sensitivity — just a single MHC molecule loaded with antigen can trigger the elimination of the infected cell (28). Taken together, these findings suggest that HIV-specific CD8 T cells, critical for controlling the infection, may be harnessed to target and eradicate the latent reservoir.

In this study, we propose that Nef is expressed in latently HIV-1-infected CD4 T cells and is hence responsible, at least in part, for the defect in CD8 T cell-mediated clearance as a consequence of reduced antigen presentation. To test this idea, we investigated whether small molecule inhibitors of Nef from 2 distinct chemical classes, previously shown to bind directly to Nef and to block its activity in HIV-1-infected cell lines (29-31), could abrogate Nef-mediated MHC-I downregulation and allow autologous CD8 T cells to recognize and kill primary CD4 T cells latently infected with HIV-1. In combination with Nef blockade, we also tested whether prior expansion of HIV-specific CD8 T cells, as has been reported (32), could augment elimination of these latently HIV-1-infected cells.

\section{Results}

Experimental overview and generation of latently HIV-1-infected primary CD4 T cells in vitro. We asked whether antagonizing Nef activity in primary latently HIV-1-infected CD4 T cells could enhance their elimination by autologous HIV-specific CD8 $\mathrm{T}$ cells as a result of impairing Nef-mediated MHC-I downregulation. To test this hypothesis, we employed the use of small molecule inhibitors previously shown to bind HIV-1 Nef directly and block its functions related to HIV-1 infectivity and replication enhancement (29-31). The structures of the 4 inhibitors used in this study are shown in Supplemental Figure 1 (supplemental material available online with this article; https://doi.org/10.1172/jci.insight.93684DS1). Inhibitors DQBS and B9 were added to latently infected cell cultures at $5 \mu \mathrm{M}$ each, whereas B9 analogs JZ-96-21 and JZ-97-21 were used at a final concentration of $0.5 \mu \mathrm{M}$ each. The inhibitor concentration employed for compound B9 is based on the $\mathrm{IC}_{50}$ for inhibition of Nef-mediated Src-family kinase activation and HIV-1 infectivity enhancement in vitro (29), whereas DQBS was tested at $5 \mu \mathrm{M}$ due to its ability to prevent MHC-I downregulation at this concentration in Nef-transfected cells (30). JZ-96-21 and JZ-97-21 are orally bioavailable, non-azo derivatives of compound B9 with reduced cytotoxicity and are active at the indicated concentrations in HIV-1 replication assays (ref. 31 and data not shown). We also tested whether prior expansion of CD8 T cells with HIV-1 peptides would augment any observed effects as previously reported $(32,33)$. CD8 T cell-mediated recognition of latently HIV-1-infected targets was characterized in an IFN- $\gamma$ release assay. Additionally, we determined the impact of Nef blockade on the direct killing of latently infected CD4 T cells by HIV-specific CD8 T cells utilizing a viral outgrowth assay. A schematic for the assays performed is provided in Figure 1.

We adapted a previously characterized CCL19 chemokine-dependent model (34-36) to generate primary latently HIV-1-infected CD4 T cells in vitro. Subject characteristics of all HIV-1-infected donors are provided in Supplemental Table 1. Our model differed in that we sorted resting CD4 T cells (i.e., those not expressing CD25, CD69, or HLA-DR) 3 days after infection with competent HIV-1 rather than prior to infection so that any artifacts of viral activation that may have led to T cell activation would be minimized. We added HAART (3TC, tenofovir, and nevirapine) to the cultures following 24 hours of infection to ensure a single-round infection. Another modification to the protocol was the use of magnetically labeled 


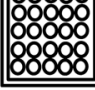

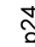
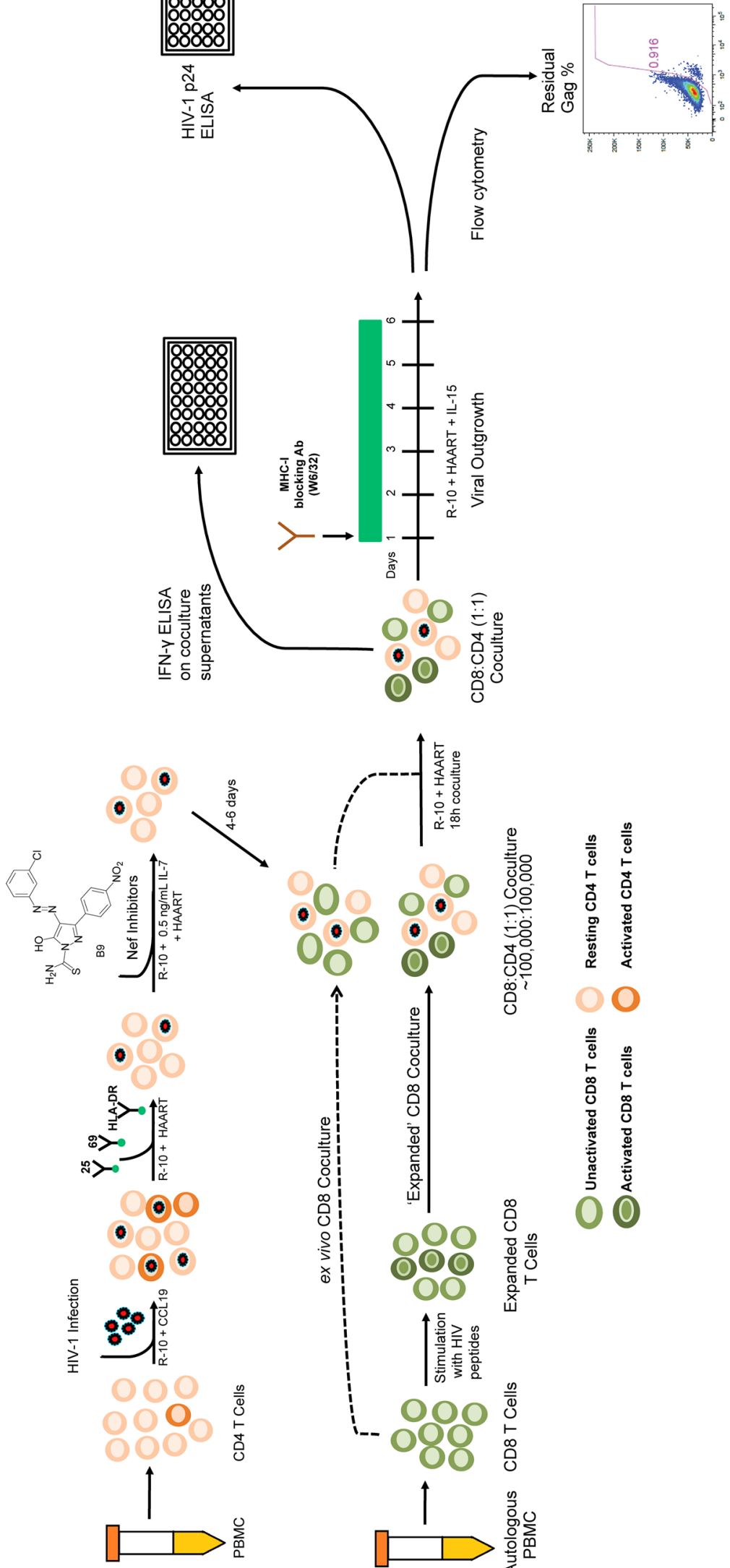

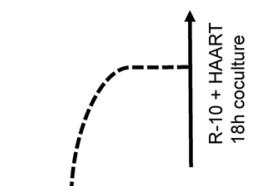

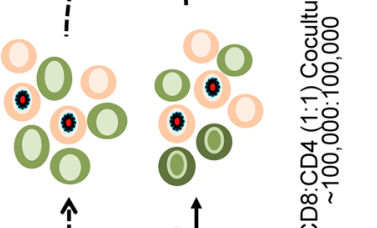

0
$\stackrel{0}{3}$
$\bar{z}$
0
0
0
0
0
$\frac{0}{0}$
$\frac{0}{0}$
$\frac{0}{0}$
$\bar{x}$

000 ปั 00

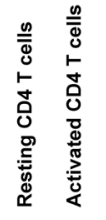

000

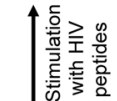

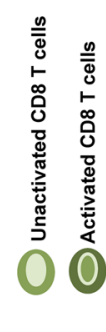

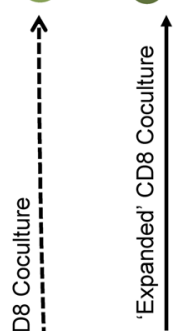

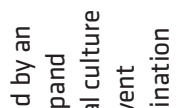

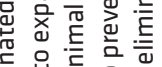

듬 은 를 웧

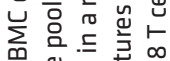

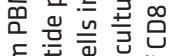

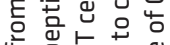

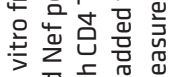

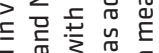

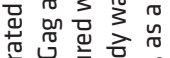

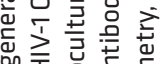

an

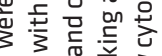

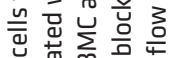

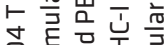

焉㟧立

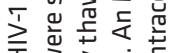

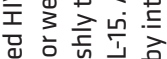

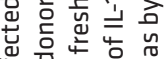

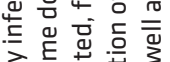

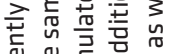

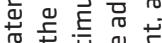

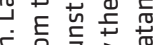

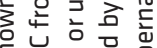

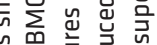

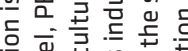

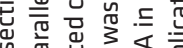

红

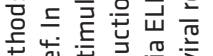

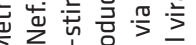

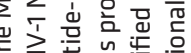

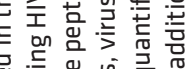

पू山

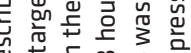

嗐

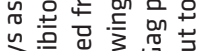

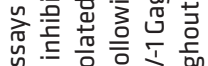

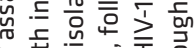

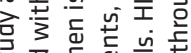

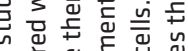

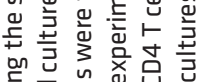

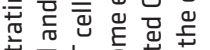

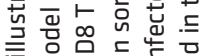

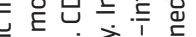

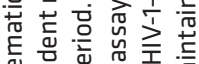

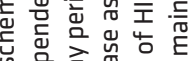

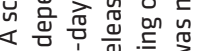

ง

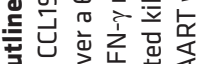

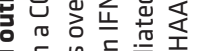

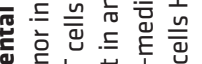

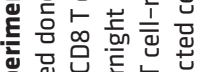

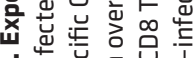

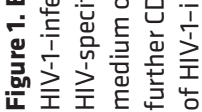


virus to increase the frequency of infected CD4 T cells in our assay as described previously (36-38). We cultured our cells in $0.5 \mathrm{ng} / \mathrm{ml} \mathrm{IL-7} \mathrm{to} \mathrm{promote} \mathrm{survival} \mathrm{of} \mathrm{the} \mathrm{CD4} \mathrm{T;} \mathrm{at} \mathrm{this} \mathrm{low} \mathrm{dose,} \mathrm{IL-7} \mathrm{did} \mathrm{not}$ augment the expression of surface activation markers (Supplemental Figure 2). Recently, Jones et al. utilized a similar in vitro latency model to generate primary latently HIV-1-infected CD4 T cells and studied HIV-specific CD8 responses against these cells (36). Validation of the in vitro latency model is presented in Figure 2A, whereby primary CD4 T cells were infected with HIV-1 viruses NL4-3 (CXCR4 tropic) or J32228M4 (CCR5 tropic). These 2 viruses were used for the IFN- $\gamma$ release assay with ex vivo CD8 T cells, whereas all other experiments were performed with NL4-3 and the NL4-3 virus lacking Nef expression (NL4-3 $\Delta \mathrm{Nef}$ ). As shown in Figure 2A, when adjusted for the mock-infected cells background, less than $1 \%$ of exogenously infected resting CD4 T cells expressed HIV-1 Gag p24 in medium alone versus $8.4 \%$ and $9.2 \%$ in response to IL-15 stimulation with NL4-3 and J32228M4 virus infections, respectively. The increase in frequency of HIV-1 Gag ${ }^{+}$cells following IL-15 stimulation in the presence of HAART indicates that $\sim 10 \%$ of the CD4 T cells were latently infected with either virus. Summary data from 8 individual experiments generating primary latently infected CD4 T cells with the $2 \mathrm{HIV}-1$ viruses J32228M4 $(n=2)$ and NL4-3 $(n=6)$ are also depicted in Figure 2A. Overall, HIV-1 Gag cell median frequency was $1.7 \%$ in medium alone compared with median $6.2 \%$ cells stimulated with IL-15 for 6 days in HAART $(P=0.008)$. Few HIV-1 Gag ${ }^{+}$cells are detected in infected cultures without any stimulation, and such HIV-1 Gag-producing resting HIV-1-infected CD4 T cells have previously been reported (39). Furthermore, such resting HIV-1 Gag+ T cells are also detected in vivo in the PBMC of donors on suppressive HAART (9-11). All experiments conducted in this study were therefore performed on latently HIV-1-infected CD4 T cells from various donors generated by this protocol.

To confirm that the HAART cocktail utilized in our experiments - composed of the nucleoside reverse transcriptase inhibitors (NRTI) 3TC and tenofovir and the nonnucleoside reverse transcriptase inhibitor (nNRTI) nevirapine - could effectively block new rounds of infection, we infected primary CD4 T cells with HIV-1 NL4-3 in the presence or absence of HAART for 5 days (Figure 2B). For this experiment only, CD4 T cells were stimulated with anti-CD3 and anti-CD28 antibodies and IL-2 for 2 days prior to infection to support robust HIV-1 infection (38). Infection of activated CD4 T cells, which are more susceptible to HIV-1 infection than resting CD4 T cells, was abrogated in the presence of HAART. Adjusted for mock-infected cells, $6.6 \%$ of CD4 T cells were HIV-1 Gag ${ }^{+}$in the absence of HAART, whereas HIV-1 Gag ${ }^{+}$cells were not detected in cultures treated with HAART. These results indicate that HAART employed in our study effectively blocked infection of CD4 T cells.

We next tested the ability of the Nef inhibitors to impair Nef-mediated MHC-I downregulation in our in vitro latency model. Resting CD4 T cells were latently infected with HIV-1 NL4-3 and then cultured with each of the Nef inhibitors at the indicated concentrations for 4 days, and surface MHC-I expression was assessed by flow cytometry, as shown in Figure 2C. We observed a restoration of MHC-I expression only on latently HIV-1-infected CD4 T cells and no modulation of MHC-I on uninfected cells following Nef blockade, measured as the fold increase of the mean fluorescence intensity (MFI) of MHC-I between the inhibitor-treated $\mathrm{Gag}^{+}$cells and DMSO control Gag${ }^{+}$cells. The MFI is indicative of the number of molecules of MHC-I expressed on a single cell. Finally, we determined the ratio of MHC-I MFI between the HIV-1 Gag-expressing $\left(\mathrm{Gag}^{+}\right)$and the Gag- CD4 T cells within each treatment in Figure 2D. The DMSO control cells exhibit maximal MHC-I perturbation (MHC-I MFI ratio of 0.56) compared with treatment with each of the inhibitors; the median ratio 0.8 is indicative of restoration of MHC-I molecules on the surface of resting HIV-1-infected CD4 T cells. Relative to the paired DMSO control, Nef inhibitors individually restored MHC-I expression between the $\mathrm{Gag}^{+}$and $\mathrm{Gag}^{-}$subpopulations within samples by $48 \%$ (DQBS), 38\% (B9), 63\% (JZ-96-21), and 38\% (JZ-97-21). Overall, these results indicate that the inhibitors disrupt Nef-mediated MHC-I downregulation in an HIV-dependent manner.

Nef inhibition enhances the recognition of autologous latently HIV-1-infected primary CD4 T cells. Resting HIV-1-infected or mock-infected CD4 T cells from various HIV-1-infected donors were generated as per the protocol described above. Small molecule inhibitors of HIV-1 Nef were added to latently infected cells at the indicated concentrations for a period of 4-6 days. Following this duration, CD4 T cells were washed and cocultured in a 1:1 ratio with autologous ex vivo or HIV-1 peptide-stimulated CD8 T cells for 18 hours in an IFN- $\gamma$ release assay. IFN- $\gamma$ was quantified in the supernatants via ELISA following overnight coincubation. Figure 3A is an example of an experiment of coculture of ex vivo autologous CD8 T cells with mock and latently HIV-1-infected CD4 T cells. We compared IFN- $\gamma$ detected in the 
A
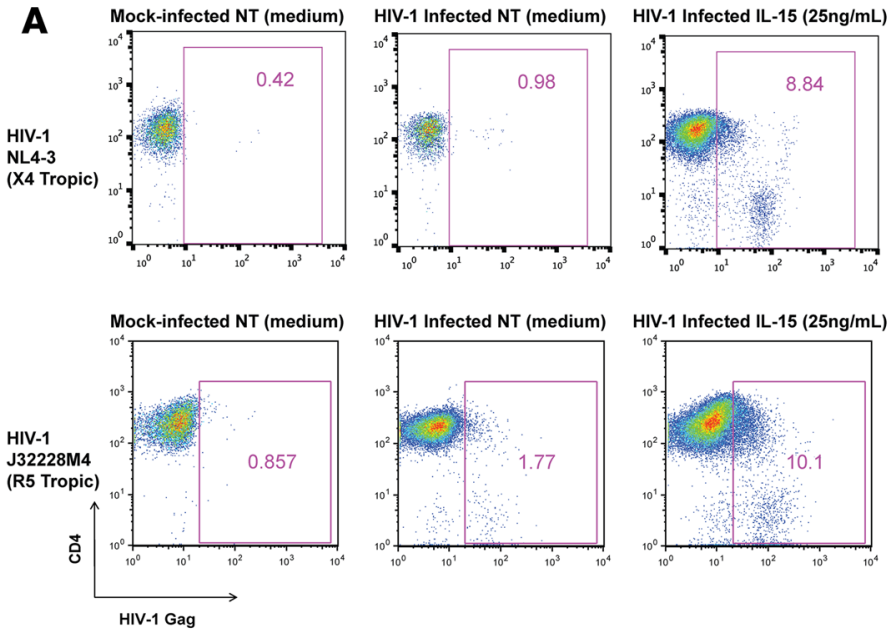

B
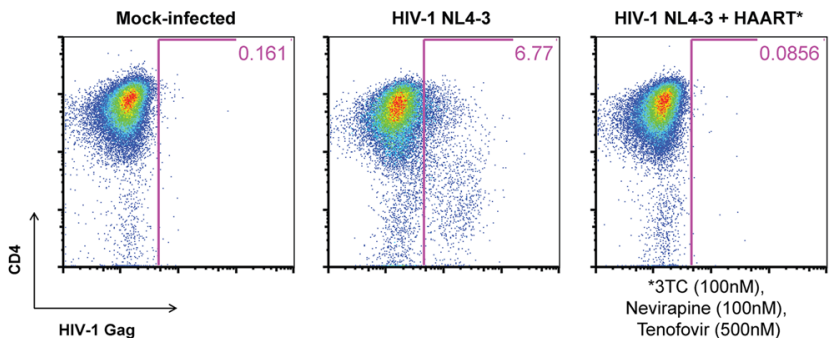

C

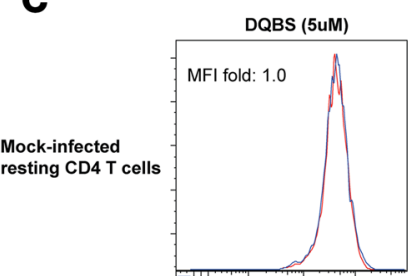

$\underline{\text { Inter-sample MH }}$
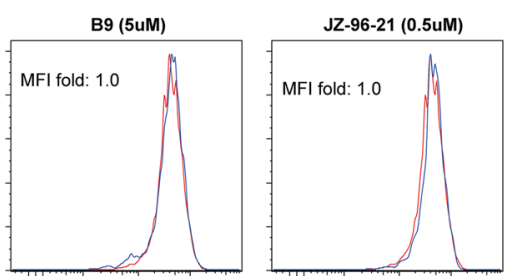

MFI Fold $=\underline{\text { MFI Inhibitor }}$
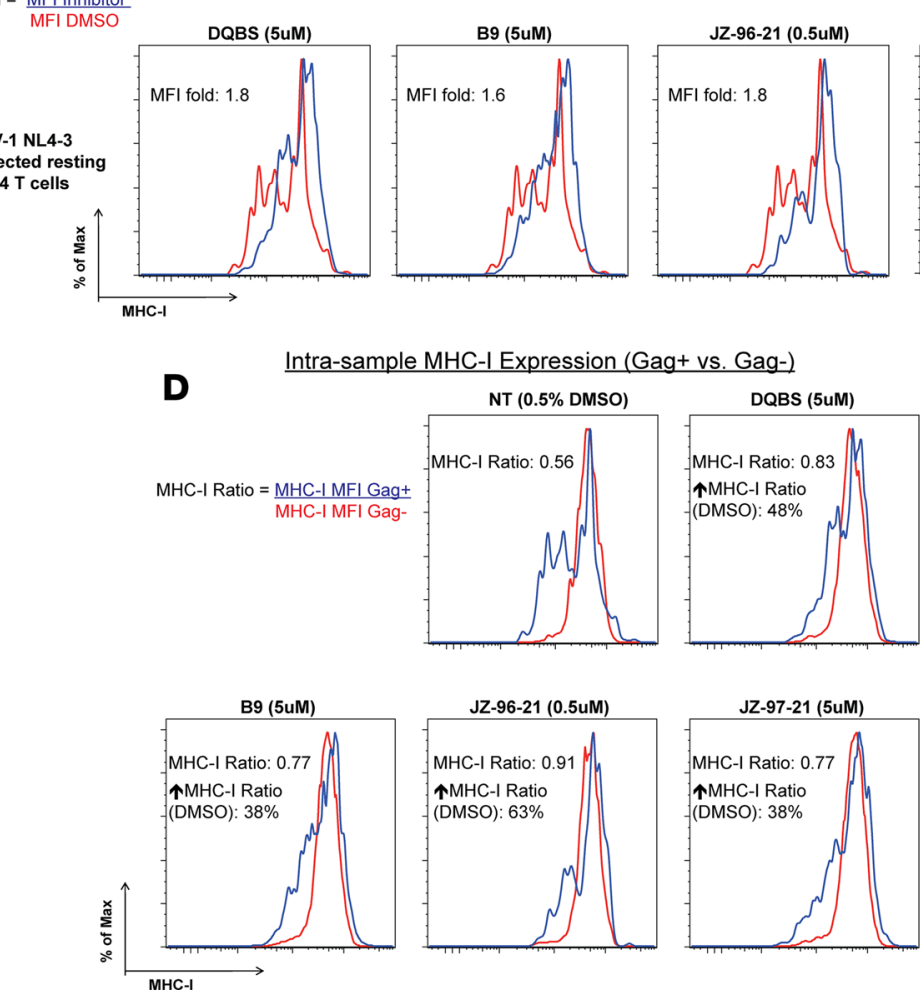
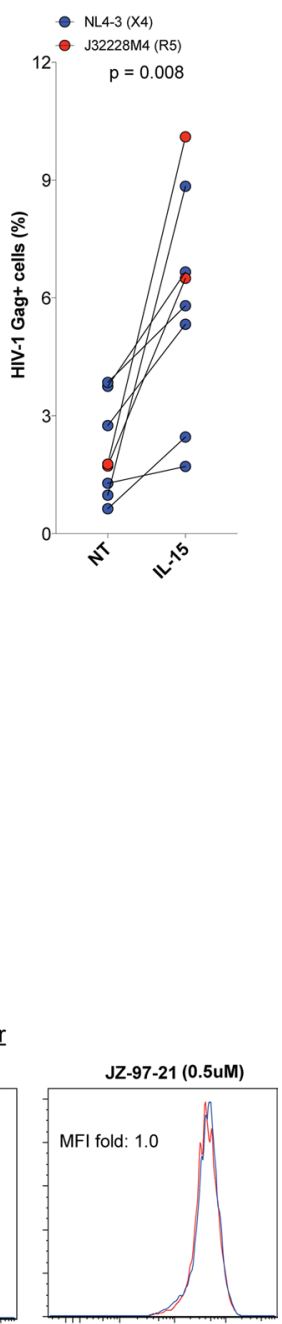

Figure 2. Validation of the primary CD4 T cells in vitro latency model. (A) CD4

T cells from an HIV-1-uninfected donor were exogenously infected with $\mathrm{X} 4$ tropic HIV-1 NL4-3 (top panel) or an R5 tropic primary HIV-1 isolate J32228M4 (bottom panel) to generate latently infected cells as described in the Methods. Latently HIV1-infected cells were cultured in medium alone or with IL-15 for 6 days to induce virus production in the presence of HAART. HIV-1 Gag p24-producing cells were enumerated by flow cytometry. Representative flow cytometry plots and summary data from 8 experiments are shown; Wilcoxon matched pairs tests were performed to determine statistical significance.

Latently infected CD4 T cells generated via this method were utilized for all further experiments. (B) Primary CD4 T cells were isolated and stimulated with anti-CD3 and anti-CD28 antibodies ( $1 \mu \mathrm{g} / \mathrm{ml}$ each) and $100 \mathrm{U} / \mathrm{ml} \mathrm{IL-2}$ for 2 days and subsequently infected with HIV-1 NL4-3 in the presence or absence of the HAART cocktail used in the study. The frequency of HIV-1-infected cells was determined via flow cytometry 5 days later. Cells receiving antiretrovirals were not permissive to infection. (C) Resting HIV-1-infected (bottom panel) or mock-infected (top panel) CD4 T cells were treated with each of the Nef inhibitors for a period of 4 days in medium with HAART and $0.5 \mathrm{ng} / \mathrm{ml} \mathrm{IL-7.} \mathrm{Surface}$ MHC-I expression was then analyzed via flow cytometry. The mean fluorescence intensity (MFI) of MHC-I, indicative of the molecules of MHC-I on the surface of CD4 $T$ cells, for total mock-infected and only the HIV-1 Gag-expressing latently HIV-1infected cultures (bottom panel) were determined. The MHC-I MFI ratio between the DMSO control (red lines; NT DMSO) to those receiving Nef inhibitors (blue lines) is reported as the MFI fold (MFI Inhibitor/ MFI DMSO). Nef blockade did not alter MHC-I expression on uninfected cells. (D) Histograms depicting the MHC-I expression of the Gag ${ }^{+}$and Gag ${ }^{-}$cells within the same culture with each inhibitor and DMSO control are shown and reported as the MHC-I ratio (MFI Gag /MFI Gag-). Also shown is the percentage increase in the MHC-I ratio following Nef blockade relative to the DMSO control. Inhibitor treatment restored the $\mathrm{MHC}-\mathrm{I} \mathrm{MFI}$ ratio to a greater degree relative to the DMSO control samples. Overall, these results indicate that all 4 compounds restored $\mathrm{MHC}-\mathrm{I}$ expression on latently HIV-1-infected CD4 T cells. 
A

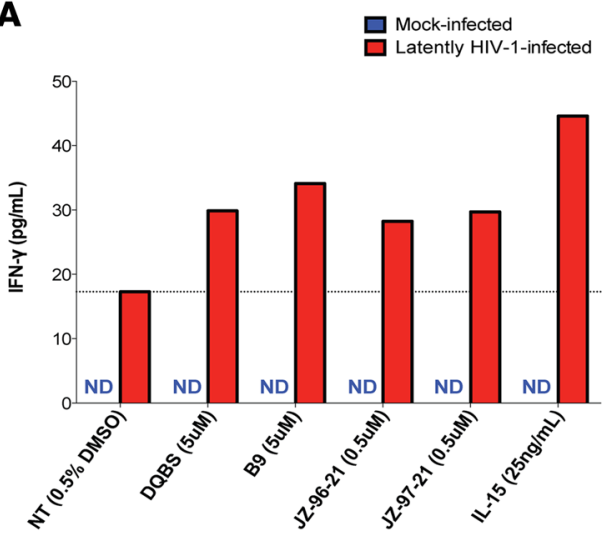

B Ex vivo $\mathrm{CD} 8$ coculture with resting mock-infected CD4 T cells

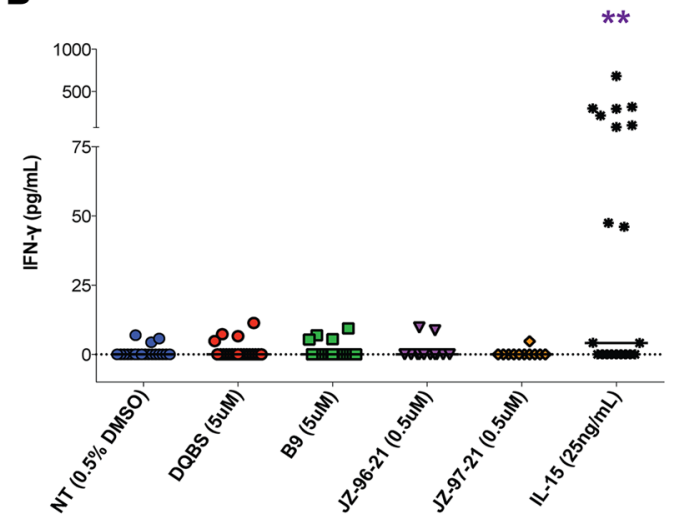

C

Ex vivo CD8 coculture with resting HIV-1-infected CD4 T cells
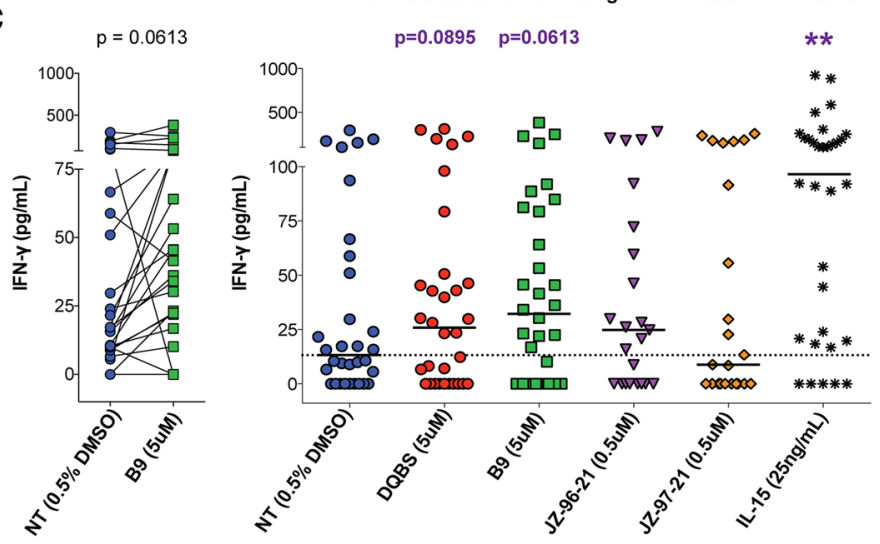

D

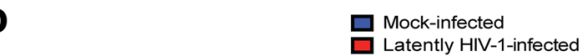

E

Expanded CD8 coculture with resting mock-infected CD4 T cells

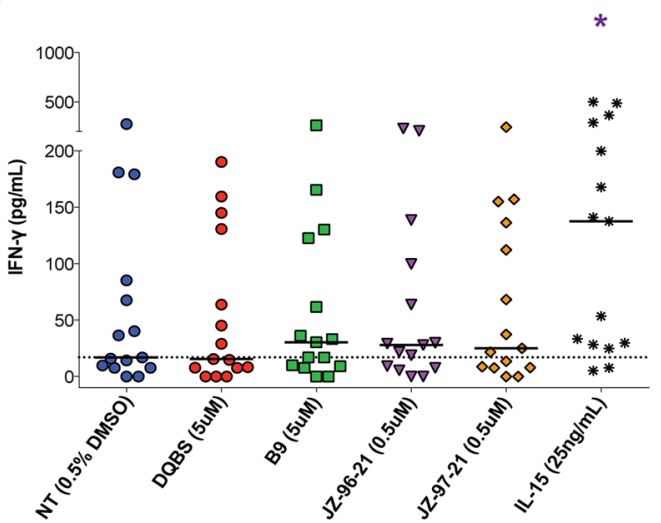

E

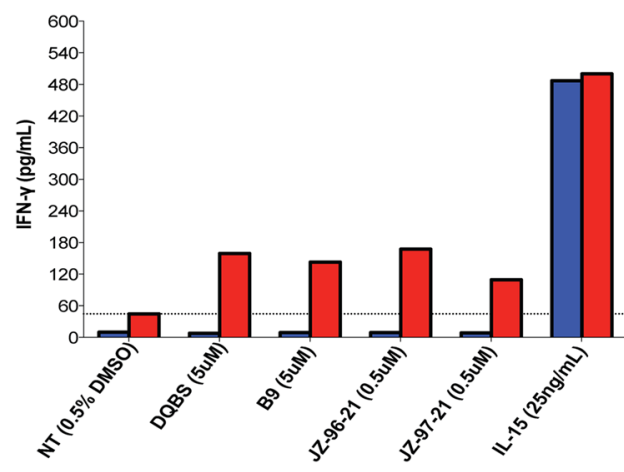

Expanded CD8 coculture with resting HIV-1-infected CD4 T cells
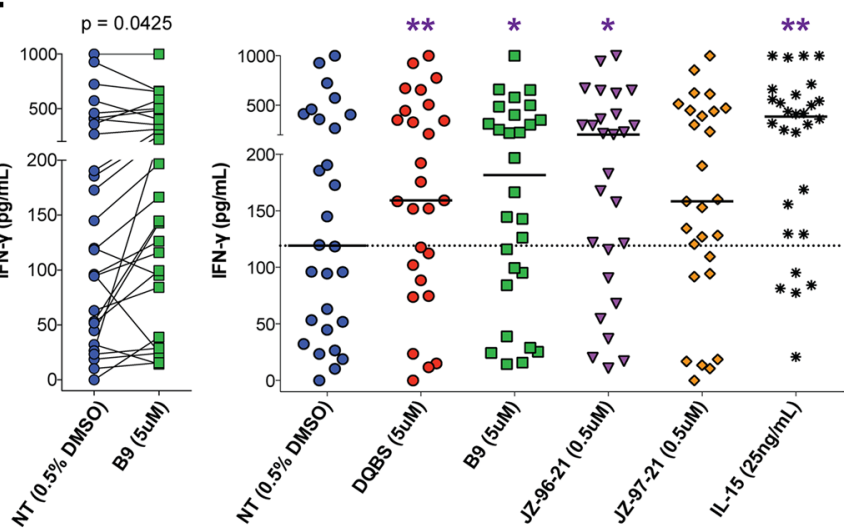

G Ex vivo CD8 coculture with resting HIV-1 ( $\triangle \mathrm{Nef}$ ) infected CD4 T cells

H Expanded CD8 coculture with resting HIV-1 ( $\triangle$ Nef) infected CD4 T cells
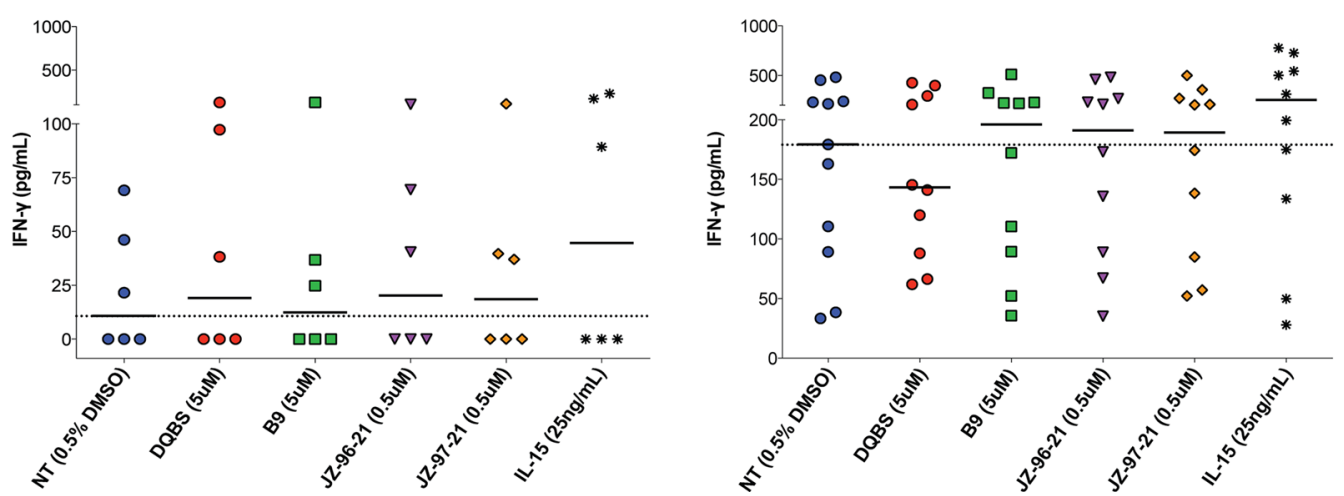
Figure 3. Nef blockade enhances CD8 recognition of latently HIV-1-infected CD4 T cells. Resting HIV-1-infected or mock-infected CD4 T cells were treated with Nef inhibitors as indicated. Ex vivo (A-C) or HIV-1 peptide-expanded autologous CD8 T cells (D-F) were cocultured overnight with latently infected cells at a 1:1 ratio. IFN- $\gamma$ was quantified in $\mathrm{pg} / \mathrm{ml}$ via ELISA. Cocultures with Nef inhibitor-treated CD4 T cells resulted in greater IFN- $\gamma$ secretion by the CD8 T cells, indicating improved recognition of latently HIV-1-infected cells compared with DMSO control. (A) Example of an individual experiment using ex vivo CD8 T cells. Summary data of ex vivo CD8 T cell cocultures with mock (B) and latently HIV-1-infected (C) CD4 T cells are shown. Paired data obtained with inhibitor B9 of ex vivo CD8 T cell cocultures with latently infected targets are shown in C. (D) An example of an individual experiment of cocultures with HIV-1 peptide-expanded CD8 T cells. E and $\mathbf{F}$ are summary data of cocultures with expanded CD8 T cells against mock- and latently HIV-1-infected CD4 T cells, respectively. Paired data depicting IFN- $\gamma$ release of expanded CD8 T cell cocultures between the control and B9 inhibitor-treated cells are shown in F. Summary data of Nef deficient (NL4-3 $\Delta$ Nef) cocultures with ex vivo (C) or autologous expanded CD8 T cells (H) are reported. No significant differences were observed between the inhibitors and control conditions in these experiments. The dotted horizontal line in each plot indicates the median DMSO control IFN- $\gamma$. Each data point represents an individual experiment. All cocultures contained HAART. (A-H) Medians shown; Wilcoxon matched-pairs tests were performed between no inhibitor (NT DMSO) and other wells to determine statistical significance. (B) $n=20$ DQBS, B9, IL-15; $n=12$ others. (C) $n=32$ for DQBS, B9, IL-15; $n=23$ others. (E) $n=15$. (F) $n=27$ DQBS, JZ-96-21, JZ-97-21; $n=28$ others. (C) $n=6$. (H) $n=11$ for NT; $n=10$ for other conditions. ND, Not detected. Statistical analysis was adjusted for multiple comparisons using the Benjamini-Hochberg procedure. ${ }^{*} P<0.05,{ }^{*} P<0.01$.

supernatants between the DMSO control (NT, i.e. no inhibitor treatment) and inhibitor-treated conditions or IL-15-stimulated cells that served as a positive control. Since IL-15-stimulated CD4 T cells maximally induce virus antigen from infected CD4 T cells (Figure 2A), we postulate that this would result in maximal CD8 $\mathrm{T}$ cell stimulation. IFN- $\gamma$ levels quantified in the supernatants of cocultures of ex vivo CD8 T cells with mock-infected (Figure 3B; $n=12-20$ ) or latently HIV-1-infected CD4 T cells (Figure 3C; $n=$ $23-32$ total; $n=10 \mathrm{~J} 32228 \mathrm{M} 4$ and $n=22 \mathrm{NL} 4-3$ infected CD4 T cells) are shown. IL-15 stimulation alone has been demonstrated to induce IFN- $\gamma$ production in T cells (40) and contributed to the significantly elevated IFN- $\gamma$ levels detected in mock-infected cocultures (Figure 3B) relative to the DMSO control. In the cocultures of resting HIV-1-infected CD4 T cells and ex vivo autologous CD8 T cells (Figure 3C), we observed a trend toward increased IFN- $\gamma$ release in conditions receiving the Nef inhibitors DQBS (median $25.8 \mathrm{pg} / \mathrm{ml}, P=0.0895$ ) and B9 (median $32.2 \mathrm{pg} / \mathrm{ml}, P=0.0613$ ) relative to the no inhibitor (DMSO) control (median $13.2 \mathrm{pg} / \mathrm{ml}$ ). Note that these changes reflect the overall donor population; responses of individual donor cells to Nef inhibitors were quite remarkable in some cases; for example, we observed an enhancement of IFN- $\gamma$ production relative to the control in 19 of 32 cocultures with the inhibitor B9.

The lack of detectable IFN- $\gamma$ responses in some participants is likely due to low frequencies of HIV-specific CD8 T cells found ex vivo in the PBMC after long-term HAART (41). As has been demonstrated previously (32), prior stimulation of HIV-specific CD8 T cells with cognate peptides enhances elimination of latently HIV-1-infected CD4 T cells, in part by expanding frequencies of HIV-specific CD8 T cells through antigen-induced proliferation. Thus, we cocultured Nef inhibitor-treated latently infected CD4 T cells with autologous CD8 T cells that had undergone 6-day stimulation with HIV-1 Gag and Nef peptide pools. Expansion of HIV-specific CD8 T cells not only increases their frequency, but also improves their ability to produce cytokines and kill target cells (32). A representative example of HIV-1 peptide expansion that increased the frequency of HIV-specific CD8 T cells is shown in Supplemental Figure 3. Indeed, we detected more IFN $-\gamma$ in response to antigen alone in cocultures using ex vivo CD8 T cells from viremic donors as compared with those from individuals on suppressive HAART (Supplemental Figure 4A). These effects were not observed for IFN- $\gamma$ responses detected with cocultures using expanded CD8 T cells in response to antigen, irrespective of treatment status (Supplemental Figure 4B). Taken together, these results indicate that HIV-peptide expansion of PBMCs increased the frequency of HIV-specific CD8 T cells and improved our ability to detect IFN- $\gamma$ responses in the assays.

Coculture of resting mock-infected CD4 T cells and expanded CD8 T cells did not result in significant changes in IFN- $\gamma$ production, irrespective of the presence of Nef inhibitors, as shown in Figure 3D in an example of an experiment and in summary data for 15 donors in Figure 3E. Again, IL-15 stimulation of mock-infected CD4 T cells for 4-6 days prior to CD8 coculture led to increased IFN- $\gamma$ detection in this condition alone as a consequence of $\mathrm{T}$ cell activation (40), unlike all other treatments that were cultured in minimal medium. Cocultures of expanded CD8 T cells with latently HIV-1-infected CD4 T cells treated with DQBS (median $159.2 \mathrm{pg} / \mathrm{ml}, P=0.0100)$, B9 (181.6 pg/ml, $P=0.0425$ ) and JZ-96-21 (median 204.1 $\mathrm{pg} / \mathrm{ml}, P=0.0243$ ) significantly increased IFN- $\gamma$ secretion relative to the DMSO control (median 119.1 $\mathrm{pg} / \mathrm{ml}$ ) shown in Figure 3F ( $n=27-28$ total; $n=10 \mathrm{~J} 32228 \mathrm{M} 4$ and $n=18$ NL4-3 latently infected CD4 T cells). Specifically, as shown in Figure 3F, cocultures of B9-treated latently infected cells and HIV-1 peptide-expanded CD8 T cells resulted in an improvement of responses from 20 of 28 experiments. A positive 
trend toward the IFN- $\gamma$ production against JZ-97-21 receiving latently infected targets was also observed (median $158.4 \mathrm{pg} / \mathrm{ml} ; P=0.1001$ ). Of note, prestimulated CD8 T cell cocultures exhibit increased IFN- $\gamma$ production in the non-inhibitor-treated conditions compared with the baseline median of ex vivo CD8 T cell cocultures (119.1 vs. $13.2 \mathrm{pg} / \mathrm{ml}$, respectively), consistent with the expansion of HIV-specific CD8 T cells in these conditions, as reported (32). Importantly, significantly increased IFN- $\gamma$ in the Nef inhibitortreated cocultures relative to the DMSO control (NT) indicates that Nef blockade enhanced the CD8 T cell recognition of latently HIV-1-infected CD4 T cells.

To confirm that the inhibitors are Nef specific, experiments were performed with NL4-3 $\Delta$ Nef to generate latently infected cells as above. Previous studies have shown that, unlike WT HIV-1 NL4-3, infectivity and replication of the $\Delta \mathrm{Nef}$ mutant are unaffected by Nef inhibitor treatment (29-31). Coculture of ex vivo autologous CD8 T cells $(n=6)$ or HIV-1 peptide-expanded CD8 T cells $(n=10-11)$ with these NL4-3 $\Delta$ Nef latently infected CD4 T cells did not result in a significant difference between control and either of the Nef inhibitor-treated samples, as reflected in Figure 3, G and H. Of interest is that baseline levels of IFN- $\gamma$ in the no inhibitor cocultures with expanded CD8 T cells were elevated if resting CD4 T cells were infected with Nef-deficient virus relative to targets that were infected with Nef-competent virus (median $179.3 \mathrm{pg} /$ $\mathrm{ml}$ vs. $119.1 \mathrm{pg} / \mathrm{ml}$, respectively), supporting the role of Nef in preventing recognition of latently infected cells. Overall, these findings demonstrate that these inhibitors function via a Nef-dependent mechanism.

Lastly, we confirmed that the IFN- $\gamma$ detected in the supernatants was produced by CD8 T cells in response to antigen by setting up cocultures between IL-15-stimulated NL4-3-infected CD4 T cells and HIV peptide-expanded autologous CD8 T cells in the presence or absence of an MHC-I blocking antibody (Supplemental Figure 5A). IL-15-stimulated cells were used as targets, as they present maximal antigen to HIV-specific CD8 T cells. MHC-I blockade abrogated CD8 T cell recognition of infected cells, with IFN- $\gamma$ levels similar in cocultures of DMSO-treated CD4 T cells. However, in the absence of the MHC-I blocking antibody, CD8 T cells secreted a greater amount of IFN- $\gamma$ due to the effective presentation of antigen from IL-15-stimulated HIV-1-infected CD4 T cells. We also cultured ex vivo or HIV-1 peptide-expanded purified CD8 $\mathrm{T}$ cells alone in equivalent cell numbers to cocultures between autologous CD4 and CD8 T cells in several parallel experiments ( $n=24$ each, ex vivo and expanded CD8 T cells; $n=48$ total) and quantitated the IFN- $\gamma$ in the supernatant following overnight incubation. We detected basal cytokine levels in CD8 T cell-alone cultures that were significantly below levels detected if CD8 T cells were cocultured with DMSO-treated autologous latently HIV-1-infected CD4 T cell targets, highlighting that the immune responses in our assays are dependent on the addition of CD8 cytotoxic T lymphocytes (CTLs) (Supplemental Figure 5B). Collectively, these results indicate that the responses detected in our assays are MHC-I restricted and exhibit antigen recognition by HIV-specific CD8 CTLs.

Increased killing of latently HIV-1-infected CD4 T cells by autologous expanded CD8 T cells following Nef blockade. We next asked whether overnight coculture of Nef inhibitor-treated HIV-1 NL4-3 latently infected CD4 T cells with HIV-1 peptide-expanded CD8 T cells would result in improved killing of latent cells. Following the IFN- $\gamma$ release assay, cells were washed and cultured in medium containing $25 \mathrm{ng} / \mathrm{ml} \mathrm{IL}-15$ to induce viral outgrowth from CD4 T cells in the presence of HAART and $10 \mu \mathrm{g} / \mathrm{ml}$ of MHC-I blocking antibody (W6/32) to prevent further CD8 T cell-mediated killing. HIV-1 Gag p24 in the supernatants was quantified 6 days later via ELISA $(n=24-26)$ as a measure of residual latently HIV-1-infected cells following CD8 coculture capable of producing virus upon IL-15 stimulation. An example of an individual experiment is shown in Figure 4A, and paired data obtained between the control and inhibitor B9 cocultures, along with summary data, are depicted in Figure 4B. Treatment with B9 reduced the latent CD4 T cell population in 23 of 26 donors tested. Overall, HIV-1 p24 levels were significantly decreased in CD4 cultures receiving inhibitors B9, JZ-96-21, and JZ-97-21 compared with control (medians 39.0, 50.4, and 39.9 vs. $57.5 \mathrm{pg} / \mathrm{ml}$ with $P=0.0004,0.0027$, and 0.0006 , respectively) indicating that prior treatment with Nef inhibitors enhanced elimination of latently HIV-1-infected cells. DQBS treatment did not significantly decrease HIV-1 p24 output in this assay. To establish that the inhibitors function in a Nef-dependent manner, we set up similar cocultures in 6 experiments with latently infected cells generated with the Nef-deficient NL4-3 virus (NL4-3 $\Delta$ Nef) shown in Figure 4C. Relative to the control DMSO coculture, none of the inhibitors had a significant effect on HIV-1 Gag p24 output in these assays, indicating that the further decreased HIV-1 Gag p24 observed in Figure 4B was primarily due to the elimination of latently infected CD4 T cells following Nef antagonism by the inhibitors. We further demonstrated that the reduction in HIV-1 Gag p24 levels in these cocultures was CD8 CTL-dependent (Supplemental Figure 5C). 

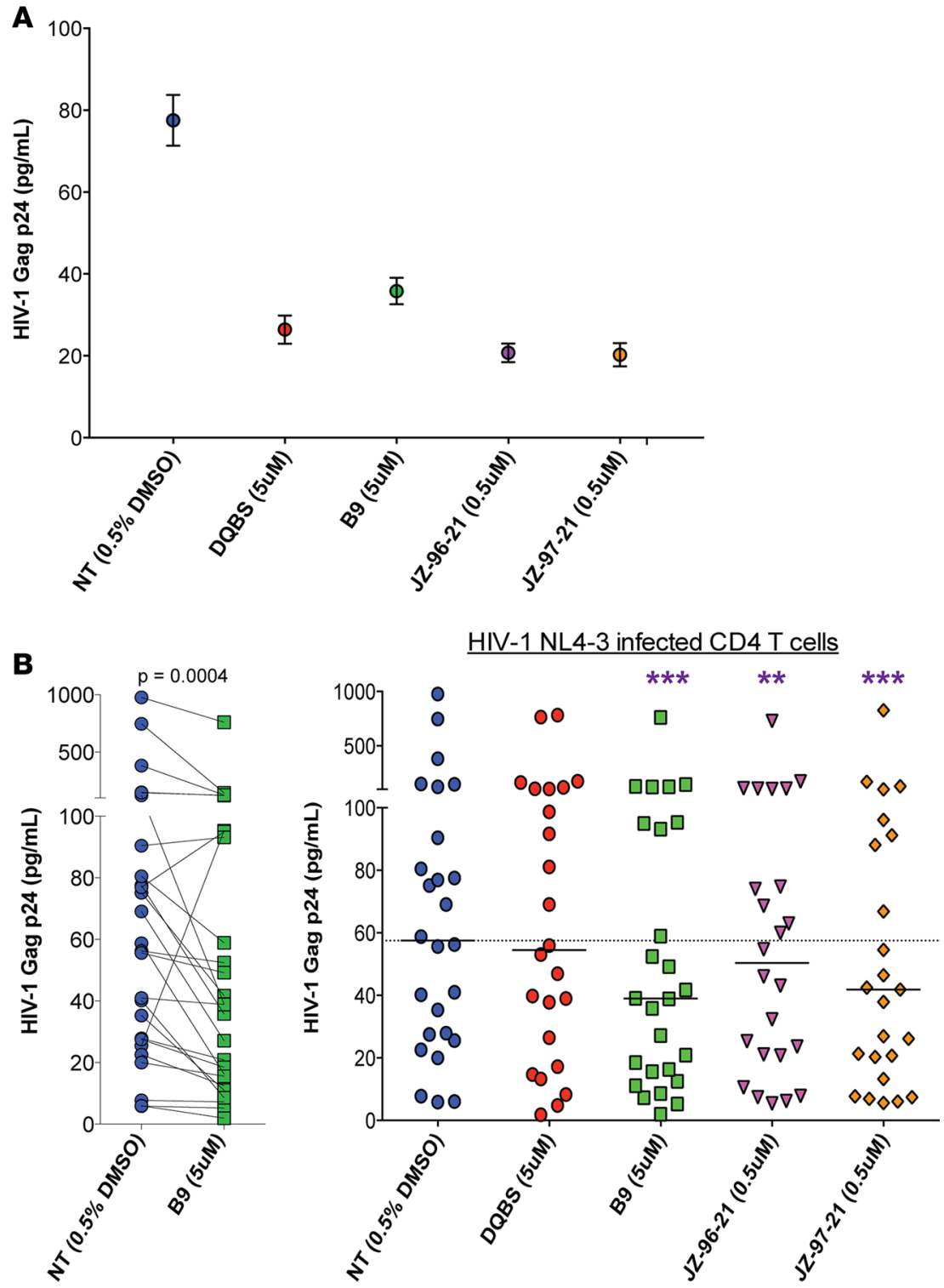

C

HIV-1 NL4-3 $\Delta$ Nef infected CD4 T cells

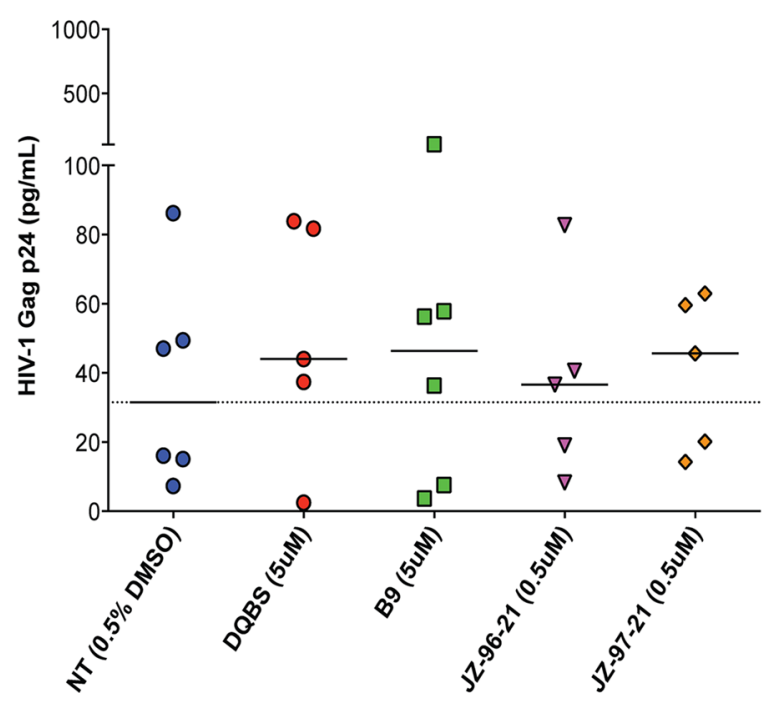

Figure 4. Improved killing of latently HIV-1-infected cells by expanded CD8 T cells following Nef blockade. Resting latently HIV-1 NL4-3-infected CD4 T cells treated with Nef inhibitors were cocultured with autologous HIV peptide-expanded CD8 T cells in coculture medium containing HAART. Virus reactivation was induced by adding IL-15 to the cultures in the presence of HAART, along with MHC-I blocking antibody W6/32 to block CD8 T cell-mediated killing for another 6 days. HIV-1 Cag p24 was quantified in the supernatants by ELISA, and an example of an experiment is shown in $\mathbf{A}$; mean and SEM of 2 replicates shown. (B) Paired data between control and inhibitor $B 9$ receiving cocultures are depicted, as well as the summary data of cocultures with each of the indicated treatments. (C) Summary data of cocultures of expanded CD8 T cells with latently infected CD4 T cells generated with Nef-deficient NL4-3 virus (NL4-3 $\Delta$ Nef) are shown. Medians shown; each symbol represents 1 individual experiment, and the dotted line indicates the median HIV-1 p24 detected in the DMSO control (NT). Wilcoxon matched-pairs tests were performed to determine statistical significance. (B) $n=24$ for DQBS, JZ-96-21, and JZ-97-21 and $n=26$ for B9 and IL-15; (C) $n=6$. Latently infected CD4 T cells were generated with HIV-1 NL4-3 virus for all data reported in $\mathbf{A}$ and $\mathbf{B}$, whereas the NL4$3 \Delta$ Nef virus was used for data in C. All $P$ values shown have been corrected for multiple comparisons using the Benjamini-Hochberg procedure. ${ }^{*} P<0.05,{ }^{* *} P<$ $0.01,{ }^{* * *} P<0.001$.

In some experiments, cells were also stained for intracellular HIV-1 Gag following viral outgrowth and analyzed via flow cytometry to quantify the frequency of HIV-1 NL4-3-infected CD4 $\mathrm{T}$ cells remaining in cultures as an alternative measure of direct killing of latently infected CD4 T cells receiving Nef inhibitors compared with control. Representative flow cytometry plots are is shown in Figure 5A, and the frequency of HIV-1 Gag cells detected in culture with inhibitor B9 and summary data are reported in Figure 5B. Similar to the ELISA results with B9, a decrease in HIV-1 p24 Gag+ cells by flow cytometry was observed in 14 of 15 donors tested. Overall, we observed significantly decreased median paired frequencies of HIV-1 Gag ${ }^{+}$cells in cocultures treated with each of the Nef inhibitors (DQBS, $0.85 \%, P=0.0031 ; \mathrm{B} 9,1.4 \%, P=$ 0.0031; JZ-96-21, 0.58\%, $P=0.0031$; JZ-97-21, $0.78 \%, P=0.0119$ relative to the DMSO control wells of $1.63 \%$ ). Reported in Figure 5C are paired data obtained from cocultures with NL4-3 $\Delta \mathrm{Nef}$ latently infected cells comparing the inhibitor B9 to DMSO controls. We did not observe a significant difference between these 2 median paired frequencies of HIV-1 Gag ${ }^{+}$CD4 T cells $(1.65 \%$ vs. $1.36 \%$, respectively; $P=0.9844)$, indicating that inhibitor action is dependent upon the presence of the Nef protein. 
To determine the degree of percent elimination observed in Figure 5, B and C, relative to the HIV-1 NL43 latently infected cell cocultures receiving only DMSO, we normalized the collective data to the control wells and report the analysis as residual $\mathrm{Gag}^{+}$cells in Figure 5D. Residual Gag ${ }^{+}$cells are defined as the frequency of HIV-1 Gag ${ }^{+}$cells as a fraction of that frequency in the corresponding DMSO control well expressed as a percentage. We show individual pairing of each experiment using inhibitor B9, as well as summary data with expanded CD8 cocultures with latently infected CD4 T cells generated with the NL4-3 and NL4-3 $\Delta$ Nef strains. Data from the Nef-competent (NL4-3, $n=14-15)$ and Nef-deficient (NL4-3 $\Delta$ Nef; $n=8-10$ ) NL4-3 viruses are reported for these analyses. We observed improved killing of latently HIV-1-infected cells with each of the Nef inhibitors DQBS, B9, JZ-96-21, and JZ-97-21 in these assays, with mean proportions of $63.7 \%(P=0.0405), 60.3 \%(P=0.0004), 44.1 \%(P=0.002)$, and $66.6 \%(P=0.0489)$ relative to control $(100 \%)$, respectively. These results indicate that a greater frequency of latently HIV-1-infected CD4 T cells were recognized and eliminated by autologous expanded CD8 T cells following Nef blockade. Importantly, cocultures with HIV-1 NL4-3 $\Delta$ Nef latently infected CD4 T cells did not exhibit improved elimination, confirming once more that the inhibitors operate via a Nef-specific mechanism.

Lastly, in 2 separate experiments, we varied the effector (autologous expanded CD8 T cells) to target (NL43 latently infected CD4 T cells) ratio from 1:1 to 10:1 to maximize the elimination of latently infected cells. In these experiments, we also re-added the Nef inhibitors during the CD4/CD8 cocultures and extended the coculture to 3 days prior to initiating viral outgrowth as described before. Representative flow cytometry plots of one of these experiments are illustrated in Figure 6A, and combined data is shown in Figure 6B. We observed dramatically improved elimination of latently infected targets given 2 doses of inhibitors at the 10:1 effector/ target ratio (E:T ratio) compared with the 1:1 coculture experiments shown in Figure 5D; mean residual Gag cells in B9 is $8.01 \%$ vs. $60.3 \%$, in JZ-96-21 is $7.26 \%$ vs. $44.1 \%$, and in JZ-97-21 is $11.21 \%$ vs. $66.6 \%$, respectively. Of note is that, at the higher E:T ratio, we were still able to detect HIV-1 Gag ${ }^{+}$cells following viral outgrowth in non-inhibitor-treated conditions, suggesting that the greater clearance of infected cells observed in these experiments was primarily due to the second dose of inhibitors provided in vitro. These results indicate that repeated dosing with Nef inhibitors in cocultures could dramatically enhance the clearance of latently infected cells.

\section{Discussion}

Our study demonstrates a novel strategy, to our knowledge, by which autologous HIV-specific CD8 T cells can be harnessed toward the elimination of primary latently HIV-1-infected CD4 T cells following Nef blockade. We report that small molecule inhibitors targeting the HIV-1 Nef protein allow for improved immune surveillance of HIV-1-infected cells by virus-specific CD8 T cells by abrogating Nef-mediated MHC-I downregulation. We have shown that Nef blockade augmented autologous HIV-specific CD8 T cell recognition as manifested by enhanced cytokine production in response to latently HIV-1-infected cells. Cytokine production by HIV-specific CD8 T cells, however, does not necessarily correlate with direct cytotoxic killing of infected cells (42-45). Hence, we also determined whether Nef blockade of latently infected cells subsequently led to their enhanced clearance by autologous HIV-1 peptide-expanded CD8 T cells. Indeed, brief coincubation of autologous CD8 T cells with latently HIV-1-infected CD4 T cells that received a single dose of inhibitors resulted in the elimination of a greater proportion of targets compared with DMSO control. Strikingly, we observed near-complete eradication of latently infected cells with 2 doses of Nef inhibitors in vitro in 2 experiments. Lastly, our data also corroborate the notion that HIV-specific CD8 $\mathrm{T}$ cells would have to be restimulated, perhaps via therapeutic vaccination, in order to substantially eliminate latently infected CD4 T cells against latently infected cells by HIV-1 peptide-expanded autologous CD8 T cells compared with ex vivo bulk CD8 T cell cocultures, as others have reported $(32,33)$.

Recent evidence suggests that, while suppressive HAART therapy represses viral replication in the blood, the effects are not as pronounced in tissue sites such as lymph nodes and the gut, perhaps due to poor bioavailability $(6,7)$. Furthermore, viral RNA is detectable in the plasma and cells by ultrasensitive assays despite ongoing therapy (46-49) and multiply spliced HIV-1 viral msRNA such as that coding for Nef is readily detectable in the plasma of HIV-1-infected individuals with undetectable plasma viral load (12, 50), suggesting that HIV-1 latency is not a state of complete transcriptional quiescence. Additionally, intensification of standard triple HAART therapy with the integrase inhibitor raltegtravir was shown to increase episomal DNA in fully suppressed individuals, indicative of ongoing HIV-1 transcription in the setting of HAART (51). Corroborating these findings, a recent report characterized a population of HIV-1 Gag protein and RNA positive peripheral blood CD4 T cells ex vivo from HIV-1-infected individuals on suppressive HAART (11). The HIV-1 Nef protein 
A NT $(0.5 \%$ DMSO $)$

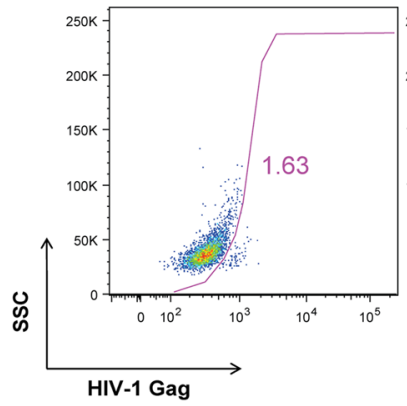

DQBS (5uM)

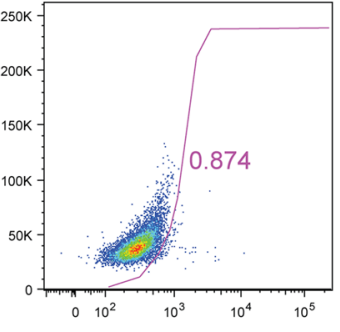

B9 (5uM)

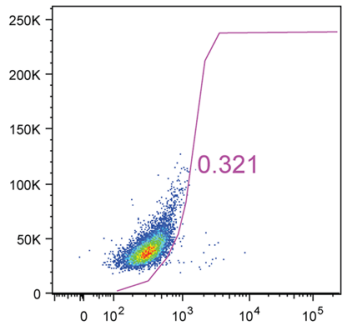

JZ-96-21 (0.5uM)

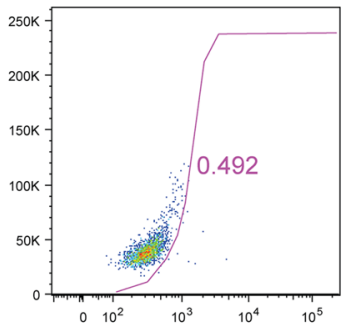

JZ-97-21 (0.5uM)

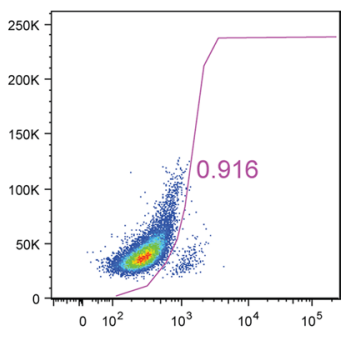

B

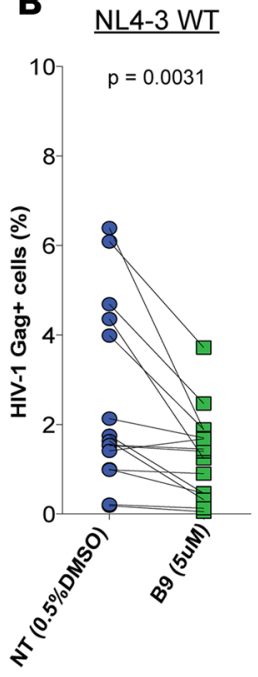

D

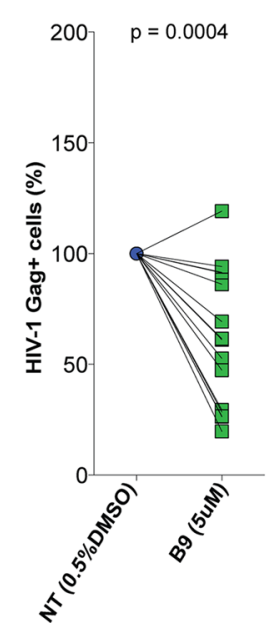

HIV-1 Gag+ CD4 T cells - NL4-3 WT virus (\%)

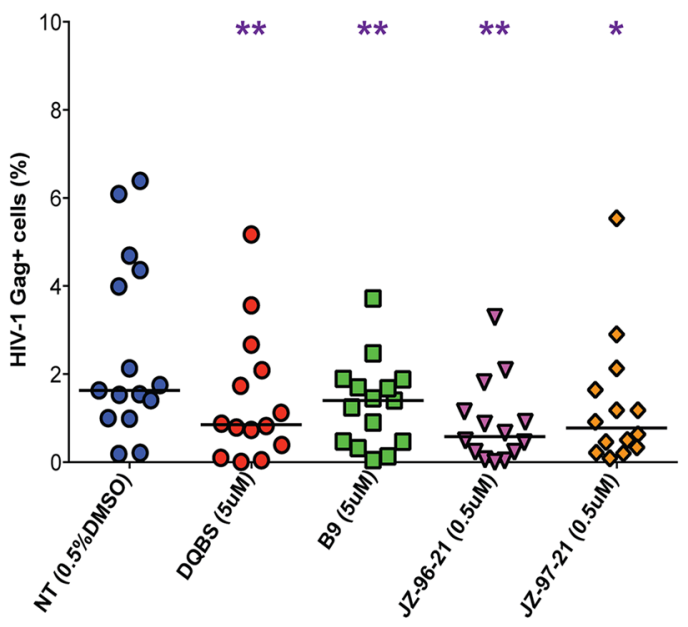

C

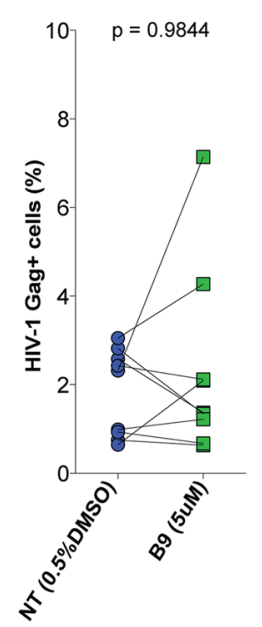

Residual Gag+ CD4 T cells (\%)

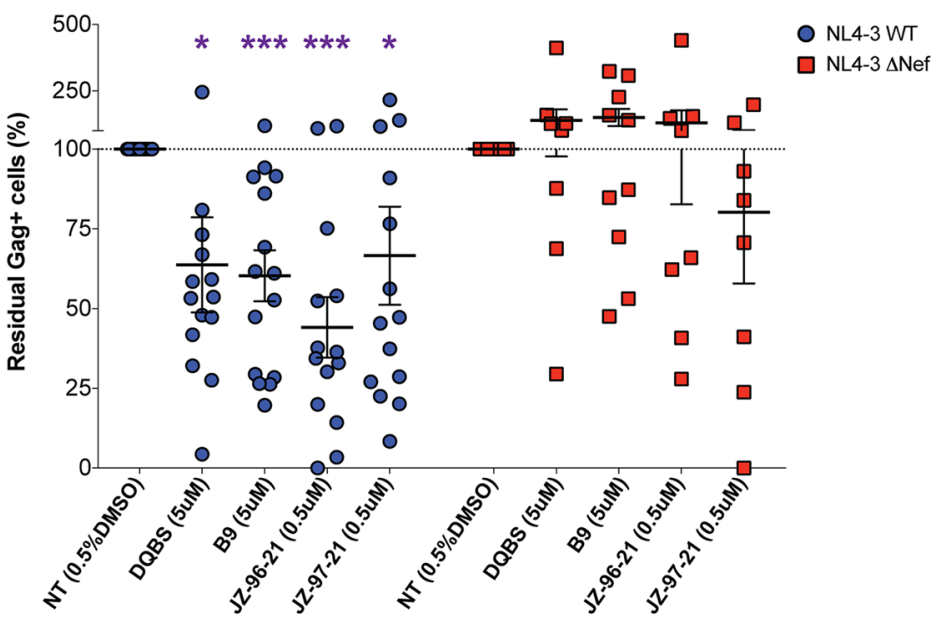

Figure 5. Nef blockade resulted in greater elimination of latently HIV-1-infected CD4 T cells. Resting latently HIV-1-infected cells were treated with Nef inhibitors for 4-6 days. Cells were then cocultured at a 1:1 ratio with autologous HIV-1 peptide-expanded CD8 T cells in medium containing HAART. One day later, an MHC-I blocking antibody, W6/32, was added to the wells to prevent further CD8 T cell-mediated killing, along with IL-15 to induce virus production. Six days later, the frequency of CD4 T cells producing HIV-1 Gag p24 were enumerated via flow cytometry. Shown in A are representative plots of an experiment with CD4 T cells infected with the NL4-3 virus. (B) Paired data between the control and B9-treated latently NL4-3-infected cell cocultures with expanded CD8 T cells are shown, and the summary data with each indicated treatment is shown. (C) Paired results between cocultures of latently NL4-3 $\Delta$ Nef-infected cells receiving the inhibitor B9 and the DMSO control are shown. (D) Data were normalized to the DMSO control condition and are reported as the Residual Gag CD4 T cells, the percentage of HIV-1 Gag p24+ cells as a fraction of the DMSO control. Paired data between control and B9 cocultures and summary data are shown. Medians shown for B and C. Mean with SEM shown for summary data in D. (B-D) NL4-3 WT: $n=15$ B9; $n=14$ others. NL4-3 $\Delta$ Nef: $n=10$ B9, $n=8$ others. All analyses were performed comparing the control (NT) data to the paired Nef inhibitor data. Wilcoxon matched-pair tests were performed to determine statistical significance in $\mathbf{B}$ and $\mathbf{C}$. Paired $t$ tests were performed to determine statistical significance for Residual Gag cells (\%) data in $\mathbf{D}$. Reported $P$ values have been corrected for multiple comparisons using the Benjamini-Hochberg procedure. ${ }^{*} P<0.05,{ }^{* *} P<0.01,{ }^{* *} P<0.001$. 

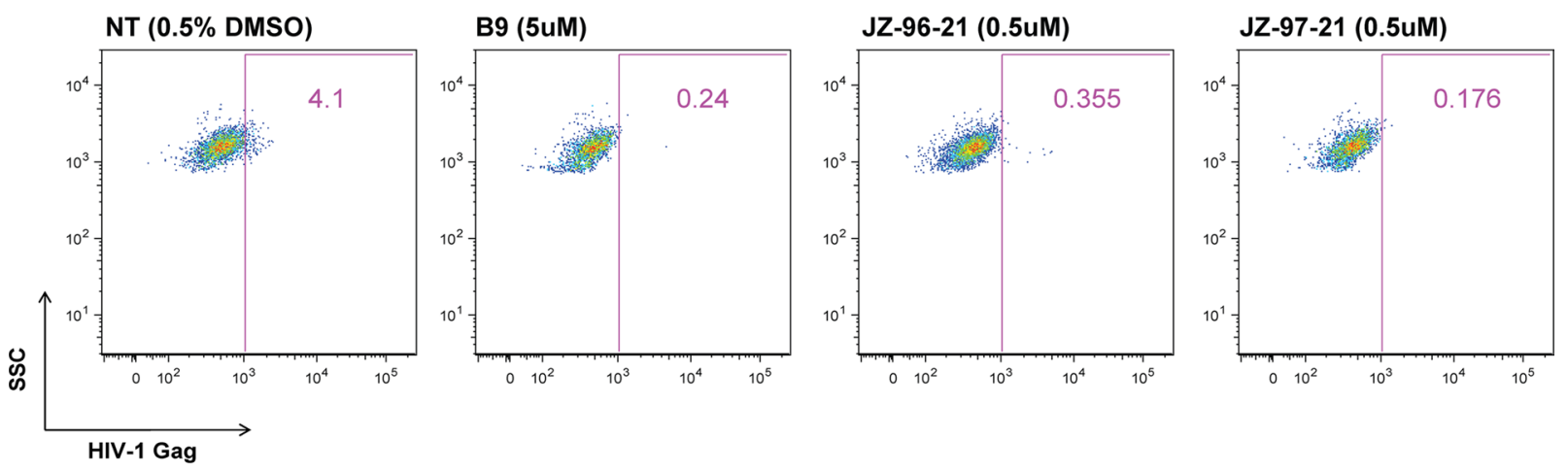

\section{B}

Residual Gag+ CD4 T cells (\%) - 10:1 CD8:CD4 + Two doses of Nef Inhibitors

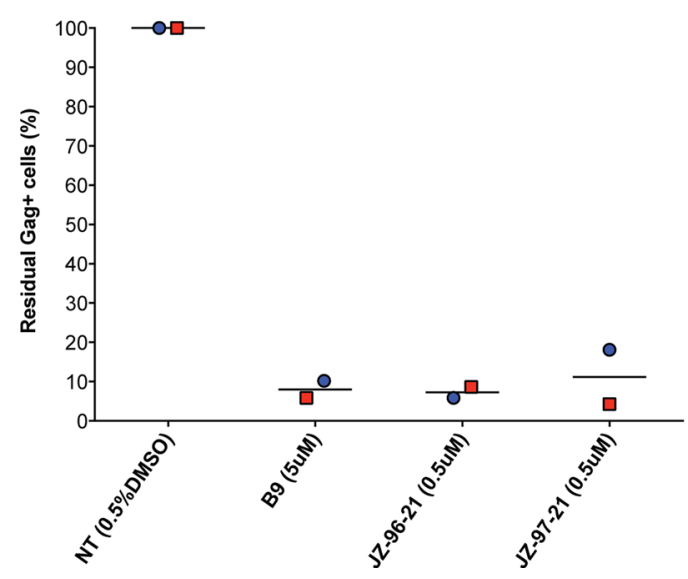

Figure 6. Dualdosing with Nef inhibitors eliminated the majority of latently HIV1 infected CD4 T cells. In 2 separate experiments, resting latently HIV-1-infected cells were treated with the indicated Nef inhibitors for 6 days, washed, and then cocultured at 10:1 CD8/CD4 ratio, with autologous HIV-1 peptide-expanded CD8 T cells in medium containing HAART. A second dose of Nef inhibitors was added to the cocultures for 3 more days. Following this period, cocultures were washed, and the viral outgrowth assay was initiated. The frequency of HIV-1 $\mathrm{Gag}^{+}$cells were enumerated 6 days later by flow cytometry. Representative data from an individual experiment is shown in $\mathbf{A}$, and Residual Gag ${ }^{+}$cells for these experiments are illustrated in B. Means shown; colors depict independent paired experiments; $n=2$.

is also detected in the PBMC of patients on HAART (13), as well as in plasma extracellular vesicles, correlating with HIV-1 pathogenesis (14). To support these findings, we too were able to detect Nef protein via Western blot analysis in the PBMC of HIV-1-infected donors, including those on suppressive HAART (Supplemental Figure 6). Recently, Imamichi et al. reported that CD4 T cells from HIV-1-infected individuals on prolonged therapy contain "defective" proviral sequences that retain open reading frames (ORFs) capable of Nef protein translation (50). Nef expression alone in transgenic mice is sufficient to exhibit AIDS-like symptoms (52, 53); hence, these defective proviruses may contribute to HIV-1 pathogenesis in the setting of HAART. Furthermore, Nef expression within clonally expanded CD4 T cells would promote immune evasion, alluding to how such HIV-1 genomes could persist indefinitely. CD8 CTL are extremely sensitive to MHC-I presentation in vivo, and it has been demonstrated that virus-mediated MHC-I downregulation dramatically diminishes the ability of CD8 T cell clones to interact with and thus eliminate infected cells, even at high antigenic concentrations as demonstrated by Halle et al. (54). In the setting of SIV, rhesus macaques infected with a Nef strain incapable of downregulating MHC-I, but not other molecules, reverts to WT early during acute infection in vivo, implicating SIV in imparting strong selective pressure for this feature of immune evasion (55). Additionally, the degree of MHC-I downregulation in vivo in SIV-infected rhesus macaques correlates with disease progression, highlighting that MHC-I downregulation confers resistance to infected cells that is indispensible to viral pathogenesis in vivo (56). This increasing body of evidence suggests that low-level Nef production during HAART promotes immune evasion of infected cells via MHC-I downregulation, facilitates persistence, and shapes the reservoir in vivo. Hence, Nef blockade would be an attractive strategy to facilitate eradication of the HIV-1 reservoir.

Latently infected cells are rare in vivo and exist at a frequency of less than 1 in $1 \times 10^{6}$ CD4 T cells for individuals on suppressive HAART $(5,57)$, making the cell numbers required to test our experiments against the natural autologous reservoir impractical. Thus, we conducted our experiments utilizing latently HIV-1-infected primary CD4 T cells generated in vitro. Various in vitro latency models have been described, each with their associated caveats, and several apply the exogenous infection of primary CD4 T 
cells with pseudotyped and modified HIV-1 strains - particularly those with the Nef gene deleted (32, 58, 59). We generated latently infected cells with WT replication-competent HIV-1 viruses, X4 tropic NL4-3, and the R5 tropic J32228M4, as these recapitulate physiological in vivo infections. The chemokine-dependent Lewin in vitro latency model we applied for our study is well characterized $(34,35,60,61)$ and its utility has been verified by other groups $(36,58)$. In this model, pretreatment of resting CD4 T cells with the CCR7 ligand CCL19 has been demonstrated to promote efficient nuclear localization and integration of HIV-1 into the genome at levels similar to CD4 T cells primed for infection with PHA stimulation $(34,60)$, recapitulating postintegration latency. Particularly, this model mimics lymphoid tissues, prominent sites for HIV-1 replication where the frequency of infected cells is greater than in PBMC (49) and the chemokine CCL19 is expressed constitutively (34), allowing for the study of HIV-1 latency, with CD4 T cells being representative of the in vivo reservoir. Other in vitro latency models such as the activation-rest based Planelles latency model and the Bcl-2 transduced CD4 T cell Siliciano model are 2 alternatives to the chemokine-dependent Lewin model we adapted in this study. With both of these models, it takes up to or over a month to generate latently infected cells unlike the Lewin model, whereby latently infected CD4 T cells are obtained within a week $(58,59)$. With the model we employed, we were able to efficiently test autologous CD8 T cell responses against latently infected CD4 T cell targets generated from several unique HIV-1-infected donors in our assays (total 41 donors as shown in Supplemental Table 1), allowing us to perform numerous independent experiments to reinforce our findings. Importantly, the Lewin model does not induce activation of CD4 T cells for HIV-1 infection, and cells are retained in a resting state representative of latently infected CD4 T cells in vivo. Still, given that the precise mechanisms by which HIV-1 established latency in vivo remains unknown, no in vitro latency model fully recapitulates in vivo latency.

Nonetheless, in vitro latency models have been instrumental in our developing understanding of immune responses against resting CD4 T cells. HIV-1 Gag-expressing resting CD4 T cells have been described in nonspreading infection (39), and importantly, these cells can be targeted for clearance by autologous CD8 T cells in in vitro assays (10). The degree to which these in vitro latently infected cells were eliminated by CD8 $\mathrm{T}$ cells inversely correlated with the size of the in vivo reservoir (10), suggesting that CD8 $\mathrm{T}$ cell-mediated in vitro elimination would be physiologically relevant if tested clinically. Recently, primary CD8 T cell-mediated recognition and elimination of autologous latently infected CD4 T cells was described in a study that had generated latently HIV-1-infected CD4 T cells identically to our approach (36), further validating our use of the Lewin model to test CD8 CTL responses following Nef blockade in our study.

Our assays tested the response of autologous bulk CD8 T cells following Nef blockade of latently infected targets that comprised multiple HIV-specific CD8 T cell clones in each coculture. Despite the emergence of multiple escape mutations, driven by CTL selection pressure, Deng et al. recently demonstrated that CD8 T cells capable of targeting autologous virus are present in the PBMC of chronically infected individuals (33), suggesting that our findings may be extended to CD8 responses against the autologous reservoir. Furthermore, we generated latently infected CD4 T cells with X4 tropic virus NL4-3 and R5 tropic virus J32228M4, and we observed similar benefits of Nef blockade, suggesting the effects are not dependent on the clade or virus tropism. Of note, significant effects are observed in our in vitro system with short-term exposure to Nef inhibitors (4-6 days) and short-term incubation with autologous CD8 T cells. Since CD8 T cells are in effect serial killers, repeated exposure may reduce the reservoir even more dramatically. In our efforts to maximize the clearance of latently infected cells, we combined a second dose of inhibitors with a boosted frequency of HIV-specific CD8 T cells in cocultures and observed the greatest degree of latent cell elimination. Additionally, the NL4-3 $\Delta$ Nef strain we utilized in several experiments is a variant of the NL4-3 virus lacking Nef expression and therefore served as the ideal control to demonstrate inhibitor specificity for the Nef protein. Intriguingly, CD8 T cell responses against $\Delta$ Nef latently infected cells were more pronounced relative to the WT virus cocultures as measured by IFN- $\gamma$ production or the elimination of resting CD4 T cells, implicating Nef in establishing HIV-1 latency via dysregulation of immune recognition. If Nef does indeed play such a role, this needs to be determined in future studies. Overall, these results set the precedent for future experiments using SIV models to determine long-term effects of CD8 T cells in the presence of Nef inhibitors and their safety in vivo. Currently, the molecules JZ-96-21 and JZ-97-21 have been shown to be orally active and well tolerated in healthy mice (31).

Current strategies to eliminate latent reservoirs, such as shock and kill, employ the use of latency reversing agents (LRAs) such as the PKC agonist Bryostatin-1 or histone deacetylase inhibitors (HDACi) romidepsin and panobinostat to induce virus production from latently infected cells in order to facili- 
tate their elimination by various mechanisms such as viral cytopathic effects or CD8 T cell-mediated clearance (62). However, to our knowledge, no single intervention to date has resulted in a significant reduction of the viral reservoir in vivo while various adverse effects have been observed $(62,63)$. Of particular concern is the observation that some LRAs could disrupt the blood brain barrier and induce hyperinflammation of neural tissue, which has been shown to correlate with HIV-1 progression $(64,65)$. Additionally, some HDACi impair HIV-specific CD8 T cell function as measured by a reduced capacity to eliminate HIV-1-infected cells (66). Hence, any HIV-1 treatment approach involving latency reversal could be associated with moderate to severe detrimental effects. Given all these caveats of the shock and kill approach, it is imperative that novel approaches such as Nef-blockade described here be tested clinically toward the development of a cure. In this study, we did not stimulate virus production from latently infected CD4 $\mathrm{T}$ cell targets prior to or during CD8 T cell coculture assays in our studies; however, we observed improved CD8 $\mathrm{T}$ cell responses following Nef inhibition in vitro. Hence, virus reactivation may not be essential to achieve clearance of viral reservoirs. Furthermore, while our results indicate that prior CD8 $\mathrm{T}$ cell expansion would greatly facilitate the recognition and the elimination of latently infected cells, it is possible that this step may not be essential in vivo either, given that CD8 T cells in lymphatic tissues receive stimulatory signals via inflammatory cytokines and/or antigen recognition (67). Indeed, in some experiments, we observed improved responses relative to control in the IFN- $\gamma$ release assay for cocultures with unstimulated ex vivo CD8 T cells. Nevertheless, an intriguing question is whether Nef blockade, in combination with safer shock and kill approaches, could facilitate reservoir reduction. Potentially, in this system, virus reactivation would induce translation of viral products, and concomitant Nef inhibition would allow for efficient antigen presentation to CTLs. Future studies will determine if shock and kill could synergize with Nef blockade.

Computational docking studies of all 4 Nef inhibitors used here (29-31) predict a common binding site at the Nef homodimer interface observed in a crystal structure of Nef in complex with a Src-family kinase SH3 domain (PDB:1EFN) (68). Since each inhibitor is predicted to interact with Nef through a shared binding site, we did not test combinations of these molecules in our assays. Nef residues predicted to be critical for binding include N126, Q104, and Q107 for B9 and its structural analogs JZ-96-21 and JZ-97-21. Mutagenesis of N126 impairs binding of these compounds in vitro. Nef N126 and Q107 are strictly conserved across all global consensus sequences belonging to multiple clades of HIV-1 in sequences obtained from the Los Alamos HIV Database, as shown in Supplemental Figure 7, supporting a key role for these residues in facilitating Nef function. These observations suggest that Nef inhibitors based on scaffolds that contact these residues may be effective against the broad range of HIV-1 subtypes responsible for the global pandemic.

In conclusion, we report an approach to facilitate the eradication of the HIV-1 latent reservoir in the setting of HAART involving small molecule antagonists of the HIV-1 Nef protein and improved CD8 T cell-mediated clearance. Our study is the first to our knowledge to report Nef blockade using novel chemical compounds with direct Nef specificity in primary latently HIV-1-infected CD4 T cells that subsequently enhanced the immune recognition and elimination by autologous CD8 T cells of resting HIV-1-infected CD4 T cells without virus reactivation. Given that the elimination of resting CD4 T cells harboring HIV-1 represents a major obstacle to the development of a cure, our proof-of-concept in vitro findings with primary cells are encouraging in this regard. However, our study tested these inhibitors using an in vitro latency model, setting the foundation for further testing of these and other Nef blockers in an in vivo model before this approach can be applied in a clinical setting. Overall, these findings lend credence to testing these and other small molecule Nef inhibitors in HIV-1 animal models to understand their potential impact on the size of the HIV-1 reservoir in vivo. Such a strategy may pave the way toward an HIV/AIDS cure.

\section{Methods}

Supplemental Methods are available online with this article.

Virus production. X4 tropic HIV-1 NL4-3 provirus plasmid and R5 tropic HIV-1 virus J32228M4 was acquired from the NIH AIDS Reagent Program (NIH). The HIV-1 NL4-3 $\Delta$ Nef proviral clone has been described elsewhere (29). HEK 293T cells (American Type Culture Collection [ATCC]) were transfected with NL4-3 and NL4-3 $\triangle$ Nef plasmids using FuGENE HD reagent (Promega). Virus supernatants were harvested 2 days following transfection and stored at $-80^{\ddagger} \mathrm{C}$ until further use. J32228M4 was propagated on primary human CD4 T cells from HIV-1-uninfected donors. Briefly, CD4 T cells were activated for 3 
days with $1 \mu \mathrm{g} / \mathrm{ml}$ each of anti-human CD3 and anti-human CD28 (clones OKT3 and 28.2, respectively; BioLegend) and $50 \mathrm{IU} / \mathrm{ml} \mathrm{IL}-2$ (Roche Diagnostics) prior to infection with J32228M4 virus. Virus was harvested 3 days following infection and stored at $-80^{\circ} \mathrm{C}$. HIV-1 p24 was quantified for each batch via ELISA (National Cancer Institute Frederick Cancer Research and Development Center, AIDS Vaccine Program).

Virus infection and in vitro latency model. PBMCs were isolated from whole blood using Ficoll-Pacque PLUS (GE Healthcare). CD4 T cells from normal and HIV-1-infected PBMCs were isolated via negative selection (StemCell Technologies). CD4 T cells were cultured at $4 \times 10^{6} / \mathrm{ml}$ in RPMI 1640 medium supplemented with 10\% FBS, $100 \mathrm{IU} / \mathrm{ml}$ penicillin, $100 \mu \mathrm{g} / \mathrm{ml}$ streptomycin, and 20mM L-glutamine (Wisent), $0.5 \mathrm{ng} / \mathrm{ml} \mathrm{IL-7} \mathrm{(R \& D} \mathrm{Systems),} \mathrm{and} \mathrm{25-100} \mathrm{nM} \mathrm{CCL19} \mathrm{(R \& D} \mathrm{Systems)} \mathrm{for} 2$ days prior to infection, modified from the Lewin model $(34-36,60)$. p24 virus $(1 \mathrm{ml}$ of $30 \mathrm{ng} / \mathrm{ml})$ was layered on $100 \mu 1$ of a 2 mM EDTA, 20\% sucrose solution in PBS (Wisent) and centrifuged at 21,000 $\mathrm{g}$ for 45 minutes at $4^{\circ} \mathrm{C}$. The virus pellet was resuspended in Viromag R/L beads (OZ Biosciences), and CD4 T cells were magnetically infected as previously reported (37). One day later, cells were washed and resuspended in medium containing HAART (100 nM Lamivudine [3TC], $100 \mathrm{nM}$ nevirapine, and $500 \mathrm{nM}$ tenofovir; NIH AIDS Reagent Program) and $0.5 \mathrm{ng} / \mathrm{ml} \mathrm{IL-7} \mathrm{in} 1 \times 10^{6} / \mathrm{ml}$. Two days later, resting CD4 T cells were isolated using magnetic beads specific to CD25, CD69, and HLA-DR antibodies conjugated to phycoerythrin (PE) as per the manufacturer's protocol (Miltenyi Biotec). Resting latently HIV-1-infected CD4 T cells were cultured in medium containing $0.5 \mathrm{ng} / \mathrm{ml} \mathrm{IL-7} \mathrm{and} \mathrm{HAART} \mathrm{in} \mathrm{a} \mathrm{96-well} \mathrm{U-bottom} \mathrm{plate} \mathrm{at} 1 \times 10^{6} / \mathrm{ml}$ and treated with individual Nef inhibitors for 4-6 days. Isolations were $>95 \%$ pure. All cells were cultured in an incubator at $37^{\circ} \mathrm{C}$ and $5 \% \mathrm{CO}_{2}$. An illustration of these procedures has been provided in Figure 1.

Nef inhibitors. Discovery and characterization of small molecule Nef inhibitors used in this study are described in detail elsewhere (29-31). All 4 compounds bind directly to Nef and inhibit Nef-dependent enhancement of HIV-1 replication and infectivity with $\mathrm{IC}_{50}$ values in the low micromolar range. These 4 inhibitors and the concentrations employed in the present study are as follows: a dihydrobenzo-1,4-dioxin-substituted analog of N-(3-aminoquinoxalin-2-yl)-4-chlorobenzenesulfonamide (DQBS; 5 $\mu \mathrm{M})$, (E)-4-([3-chlorophenyl]diazenyl)-5-hydroxy-3-(4-nitrophenyl)-1H-pyrazole-1-carbothioamide (B9; $5 \mu \mathrm{M})$, and the 2 non-azo B9 analogs JZ-96-21 (0.5 $\mu \mathrm{M}$; compound 2; ref. 31) and JZ-97-21 (0.5 $\mu \mathrm{M}$; compound 4; ref. 31). Inhibitors were dissolved in DMSO, which was diluted to a final concentration of $0.5 \%$ in cell cultures. The chemical structures of the inhibitors and a summary of published data are shown in Supplemental Figure 1.

Expansion of CD8 T cells. PBMCs were stimulated with HIV-1 consensus clade B pools for Gag (80 $\mathrm{ng} / \mathrm{ml} /$ peptide) and Nef (200 ng/ml/peptide; NIH AIDS Reagent Program) in medium containing 100 $\mathrm{IU} / \mathrm{ml} \mathrm{IL}-2$ for 6 days $(32,33)$. CD8 T cells were isolated by negative selection prior to coculture assays (StemCell Technologies).

Coculture and viral outgrowth assays. Nef inhibitor-treated, primary latently HIV-1-infected CD4 T cells were counted and washed twice in medium. Autologous CD8 T cells were isolated from peptide stimulated (see Expansion of CD8 T cells in Methods) or from freshly thawed PBMC via negative selection. Target (CD4) and effector (CD8) T cells were cocultured in 96-well U-bottom plates in $200 \mu$ of coculture medium containing HAART and $10 \mathrm{IU} / \mathrm{ml} \mathrm{IL}-2$ at a 1:1 ratio, typically $1 \times 10^{5}$ cells each. Following overnight incubation, IFN- $\gamma$ in supernatants was quantified via ELISA (BioLegend). In some experiments, following the IFN- $\gamma$ release assay, cells were resuspended in medium containing HAART and $25 \mathrm{ng} / \mathrm{ml} \mathrm{IL-15}$ (R\&D Systems) to induce viral outgrowth, along with $10 \mu \mathrm{g} / \mathrm{ml}$ of an MHC-I blocking antibody (clone W6/32, BioLegend) to prevent CD8-mediated killing during outgrowth. Six days later, cultures were analyzed for HIV-1 p24 production in supernatants via ELISA and by intracellular flow cytometry (Kc57-FITC, Beckman Coulter) as an indicator of viable HIV-1infected cells following CD8 T cell coculture. Figure 1 depicts the assays performed in the study.

Flow cytometry. Cells were stained with Live/Dead Violet (Life Technologies), anti-human CD3-PE (clone HIT3a), CD8-Alexa 700 (clone HIT8a), and CD4-APC (clone OKT4) (all from BioLegend). Anti-HIV-1 Gag p24 antibody Kc57-FITC (clone Kc57; Beckman Coulter) was used to detect intracellular HIV-1 Gag protein. Samples were run on BD FACS Calibur or BD LSRFortessa X-20. Data was analyzed on FlowJo software.

Statistics. Unless otherwise indicated, two-tailed paired-wise analyses were performed for all statistical determination between the NT - DMSO control (no inhibitor) and the other conditions. The statistical test employed has been specified in each figure legend and a $P<0.05$ after adjusting for multiple comparisons was used to determine significance and is denoted by asterisks throughout; * $P<0.05$; ${ }^{* *}, P<0.01$; ***,$P$ $<0.001)$. Tests were performed using Prism 7 software (GraphPad). All $P$ values for multiple comparisons were adjusted using the Benjamini-Hochberg procedure. 
Study approval. Informed written consent was obtained from each participant in accordance with the guidelines for conducting clinical research at the University of Toronto, St. Michael's Hospital, and Maple Leaf Medical Clinic ethics IRBs. Blood samples from HIV-1-infected and healthy HIV seronegative individuals were collected from participants at the Maple Leaf Medical Clinic and St. Michael's Hospital clinic in Toronto, Canada. All participants of the study were above the age of majority when signed consent was obtained. The IRBs approved all aspects of this study. Subject characteristics of all HIV-1-infected donors have been provided in Supplemental Table 1.

\section{Author contributions}

SM designed the research, analyzed data, and wrote the manuscript. SM, AS, AB, SF, and NA conducted experiments. FYY processed blood samples. EB, CK, and MAO recruited study participants. LAE and TES characterized the Nef inhibitors. LAE and TES reviewed and edited the manuscript. MAO designed the research, reviewed and edited the manuscript, and supervised the project.

\section{Acknowledgments}

We appreciate all the volunteers who donated blood and leukapheresis samples for this study. We thank Rupert Kaul, Tania Watts, Joel Singer, and Robert Reinhard for useful discussions. We thank A.K. Nur-ur Rahman for proofreading the manuscript. Funding for this study was provided by the Canadian Institutes of Health Research (CIHR) grant HIG-132040, the Canadian HIV Cure Enterprise Team grant HIG-133050, and the Ontario HIV Treatment Network (OHTN) AHRC grant 6769 NIAID award AI126617, co-funded by NIDA, NIMH, NINDS. SM gratefully acknowledges salary support from the QEII-GSST and CIHR graduate scholarships.

Address correspondence to: Mario Ostrowski, Room 6271, Medical Sciences Building, 1 King's College Circle, Toronto, Ontario M5S1A8, Canada. Phone: 416.946.7634; Email: mario.ostrowski@gmail.com.

1. Siliciano JD, et al. Long-term follow-up studies confirm the stability of the latent reservoir for HIV-1 in resting CD4+ T cells. Nat Med. 2003;9(6):727-728.

2. Global AIDS Update 2016. UNAIDS. http://www.unaids.org/en/resources/documents/2016/Global-AIDS-update-2016. Published May 31, 2016. Accessed August 7, 2017.

3. Chun TW, et al. Rebound of plasma viremia following cessation of antiretroviral therapy despite profoundly low levels of HIV reservoir: implications for eradication. AIDS. 2010;24(18):2803-2808.

4. Chun TW, et al. HIV-infected individuals receiving effective antiviral therapy for extended periods of time continually replenish their viral reservoir. J Clin Invest. 2005;115(11):3250-3255.

5. Crooks AM, et al. Precise Quantitation of the Latent HIV-1 Reservoir: Implications for Eradication Strategies. J Infect Dis. 2015;212(9):1361-1365.

6. Lorenzo-Redondo R, et al. Persistent HIV-1 replication maintains the tissue reservoir during therapy. Nature. 2016;530(7588):51-56

7. Fletcher CV, et al. Persistent HIV-1 replication is associated with lower antiretroviral drug concentrations in lymphatic tissues. Proc Natl Acad Sci USA. 2014;111(6):2307-2312.

8. Sigal A, et al. Cell-to-cell spread of HIV permits ongoing replication despite antiretroviral therapy. Nature. 2011;477(7362):95-98.

9. DeMaster LK, et al. A Subset of CD4/CD8 Double-Negative T Cells Expresses HIV Proteins in Patients on Antiretroviral Therapy. J Virol. 2015;90(5):2165-2179.

10. Graf EH, et al. Gag-positive reservoir cells are susceptible to HIV-specific cytotoxic T lymphocyte mediated clearance in vitro and can be detected in vivo [corrected]. PLoS ONE. 2013;8(8):e71879.

11. Baxter AE, et al. Single-Cell Characterization of Viral Translation-Competent Reservoirs in HIV-Infected Individuals. Cell Host Microbe. 2016;20(3):368-380.

12. Furtado MR, et al. Persistence of HIV-1 transcription in peripheral-blood mononuclear cells in patients receiving potent antiretroviral therapy. $N$ Engl J Med. 1999;340(21):1614-1622.

13. Wang T, et al. Intracellular Nef detected in peripheral blood mononuclear cells from HIV patients. AIDS Res Hum Retroviruses. 2015;31(2):217-220

14. Lee JH, et al. HIV-Nef and ADAM17-Containing Plasma Extracellular Vesicles Induce and Correlate with Immune Pathogenesis in Chronic HIV Infection. EBioMedicine. 2016;6:103-113.

15. Landi A, Iannucci V, Nuffel AV, Meuwissen P, Verhasselt B. One protein to rule them all: modulation of cell surface receptors and molecules by HIV Nef. Curr HIV Res. 2011;9(7):496-504.

16. Kestler HW, et al. Importance of the nef gene for maintenance of high virus loads and for development of AIDS. Cell. 1991;65(4):651-662.

17. Kirchhoff F, Greenough TC, Brettler DB, Sullivan JL, Desrosiers RC. Brief report: absence of intact nef sequences in a longterm survivor with nonprogressive HIV-1 infection. N Engl J Med. 1995;332(4):228-232.

18. Pushker R, Jacqué JM, Shields DC. Meta-analysis to test the association of HIV-1 nef amino acid differences and deletions with 
disease progression. J Virol. 2010;84(7):3644-3653.

19. Mwimanzi P, et al. Attenuation of multiple Nef functions in HIV-1 elite controllers. Retrovirology. 2013;10:1.

20. Schwartz O, Maréchal V, Le Gall S, Lemonnier F, Heard JM. Endocytosis of major histocompatibility complex class I molecules is induced by the HIV-1 Nef protein. Nat Med. 1996;2(3):338-342.

21. Mwimanzi P, Markle TJ, Ueno T, Brockman MA. Brockman, M.A., et al., Human Leukocyte Antigen (HLA) Class I Down-Regulation by Human Immunodeficiency Virus Type 1 Negative Factor (HIV-1 Nef): What Might We Learn From Natural Sequence Variants? Viruses 2012, 4, 1711-1730. Viruses. 2012;4(10):2014-2015.

22. Cohen GB, et al. The selective downregulation of class I major histocompatibility complex proteins by HIV-1 protects HIV-infected cells from NK cells. Immunity. 1999;10(6):661-671.

23. Collins KL, Chen BK, Kalams SA, Walker BD, Baltimore D. HIV-1 Nef protein protects infected primary cells against killing by cytotoxic T lymphocytes. Nature. 1998;391(6665):397-401.

24. Walker BD, Yu XG. Unravelling the mechanisms of durable control of HIV-1. Nat Rev Immunol. 2013;13(7):487-498.

25. Betts MR, et al. HIV nonprogressors preferentially maintain highly functional HIV-specific CD8+ T cells. Blood. 2006;107(12):4781-4789.

26. Buzon MJ, et al. Susceptibility to CD8 T-cell-mediated killing influences the reservoir of latently HIV-1-infected CD4 T cells. $J$ Acquir Immune Defic Syndr. 2014;65(1):1-9.

27. Ndhlovu ZM, et al. Magnitude and Kinetics of CD8+ T Cell Activation during Hyperacute HIV Infection Impact Viral Set Point. Immunity. 2015;43(3):591-604.

28. Irvine DJ, Purbhoo MA, Krogsgaard M, Davis MM. Direct observation of ligand recognition by T cells. Nature. 2002;419(6909):845-849.

29. Emert-Sedlak LA, et al. Effector kinase coupling enables high-throughput screens for direct HIV-1 Nef antagonists with antiretroviral activity. Chem Biol. 2013;20(1):82-91.

30. Trible RP, et al. Discovery of a diaminoquinoxaline benzenesulfonamide antagonist of HIV-1 Nef function using a yeast-based phenotypic screen. Retrovirology. 2013;10:135

31. Emert-Sedlak LA, et al. Synthesis and evaluation of orally active small molecule HIV-1 Nef antagonists. Bioorg Med Chem Lett. 2016;26(5):1480-1484

32. Shan L, et al. Stimulation of HIV-1-specific cytolytic T lymphocytes facilitates elimination of latent viral reservoir after virus reactivation. Immunity. 2012;36(3):491-501.

33. Deng K, et al. Broad CTL response is required to clear latent HIV-1 due to dominance of escape mutations. Nature. 2015;517(7534):381-385.

34. Saleh S, Solomon A, Wightman F, Xhilaga M, Cameron PU, Lewin SR. CCR7 ligands CCL19 and CCL21 increase permissiveness of resting memory CD4+ T cells to HIV-1 infection: a novel model of HIV-1 latency. Blood. 2007;110(13):4161-4164

35. Saleh S, et al. Expression and reactivation of HIV in a chemokine induced model of HIV latency in primary resting CD4+ T cells. Retrovirology. 2011;8:80.

36. Jones RB, et al. A Subset of Latency-Reversing Agents Expose HIV-Infected Resting CD4+ T-Cells to Recognition by Cytotoxic T-Lymphocytes. PLoS Pathog. 2016;12(4):e1005545

37. Sacha JB, Watkins DI. Synchronous infection of SIV and HIV in vitro for virology, immunology and vaccine-related studies. Nat Protoc. 2010;5(2):239-246.

38. Jones RB, et al. HERV-K-specific T cells eliminate diverse HIV-1/2 and SIV primary isolates. J Clin Invest. 2012;122(12):4473-4489.

39. Pace MJ, et al. Directly infected resting CD4+T cells can produce HIV Gag without spreading infection in a model of HIV latency. PLoS Pathog. 2012;8(7):e1002818.

40. Strengell M, et al. IL-21 in synergy with IL-15 or IL-18 enhances IFN-gamma production in human NK and T cells. J Immunol. 2003;170(11):5464-5469.

41. Ogg GS, et al. Quantitation of HIV-1-specific cytotoxic T lymphocytes and plasma load of viral RNA. Science. 1998;279(5359):2103-2106.

42. Addo MM, et al. Comprehensive epitope analysis of human immunodeficiency virus type 1 (HIV-1)-specific T-cell responses directed against the entire expressed HIV-1 genome demonstrate broadly directed responses, but no correlation to viral load. $J$ Virol. 2003;77(3):2081-2092.

43. Demers KR, Reuter MA, Betts MR. CD8(+) T-cell effector function and transcriptional regulation during HIV pathogenesis. Immunol Rev. 2013;254(1):190-206.

44. Yang H, et al. Antiviral inhibitory capacity of CD8+ T cells predicts the rate of CD4+ T-cell decline in HIV-1 infection. $J$ Infect Dis. 2012;206(4):552-561.

45. Yue FY, et al. HIV-Specific Granzyme B-Secreting but Not Gamma Interferon-Secreting T Cells Are Associated with Reduced Viral Reservoirs in Early HIV Infection. J Virol. 2017;91(8):e02233-16.

46. Palmer S, et al. Low-level viremia persists for at least 7 years in patients on suppressive antiretroviral therapy. Proc Natl Acad Sci USA. 2008;105(10):3879-3884.

47. Palmer S, et al. New real-time reverse transcriptase-initiated PCR assay with single-copy sensitivity for human immunodeficiency virus type 1 RNA in plasma. J Clin Microbiol. 2003;41(10):4531-4536.

48. Dornadula G, et al. Residual HIV-1 RNA in blood plasma of patients taking suppressive highly active antiretroviral therapy. JAMA. 1999;282(17):1627-1632.

49. Yukl SA, et al. The distribution of HIV DNA and RNA in cell subsets differs in gut and blood of HIV-positive patients on ART: implications for viral persistence. J Infect Dis. 2013;208(8):1212-1220.

50. Imamichi $\mathrm{H}$, et al. Defective HIV-1 proviruses produce novel protein-coding RNA species in HIV-infected patients on combination antiretroviral therapy. Proc Natl Acad Sci USA. 2016;113(31):8783-8788.

51. Buzón MJ, et al. HIV-1 replication and immune dynamics are affected by raltegravir intensification of HAART-suppressed subjects. Nat Med. 2010;16(4):460-465.

52. Simard MC, Chrobak P, Kay DG, Hanna Z, Jothy S, Jolicoeur P. Expression of simian immunodeficiency virus nef in immune cells of transgenic mice leads to a severe AIDS-like disease. J Virol. 2002;76(8):3981-3995 
53. Hanna Z, Kay DG, Rebai N, Guimond A, Jothy S, Jolicoeur P. Nef harbors a major determinant of pathogenicity for an AIDSlike disease induced by HIV-1 in transgenic mice. Cell. 1998;95(2):163-175.

54. Halle S, et al. In Vivo Killing Capacity of Cytotoxic T Cells Is Limited and Involves Dynamic Interactions and T Cell Cooperativity. Immunity. 2016;44(2):233-245.

55. Münch J, Stolte N, Fuchs D, Stahl-Hennig C, Kirchhoff F. Efficient class I major histocompatibility complex down-regulation by simian immunodeficiency virus Nef is associated with a strong selective advantage in infected rhesus macaques. $J$ Virol. 2001;75(21):10532-10536.

56. Friedrich TC, et al. High viremia is associated with high levels of in vivo major histocompatibility complex class I Downregulation in rhesus macaques infected with simian immunodeficiency virus SIVmac239. J Virol. 2010;84(10):5443-5447.

57. Eriksson S, et al. Comparative analysis of measures of viral reservoirs in HIV-1 eradication studies. PLoS Pathog. 2013;9(2):e1003174

58. Spina CA, et al. An in-depth comparison of latent HIV-1 reactivation in multiple cell model systems and resting CD4+ T cells from aviremic patients. PLoS Pathog. 2013;9(12):e1003834.

59. Archin NM, Sung JM, Garrido C, Soriano-Sarabia N, Margolis DM. Eradicating HIV-1 infection: seeking to clear a persistent pathogen. Nat Rev Microbiol. 2014;12(11):750-764.

60. Cameron PU, et al. Establishment of HIV-1 latency in resting CD4+ T cells depends on chemokine-induced changes in the actin cytoskeleton. Proc Natl Acad Sci USA. 2010;107(39):16934-16939.

61. Saleh S, et al. HIV integration and the establishment of latency in CCL19-treated resting CD4(+) T cells require activation of NF-кB. Retrovirology. 2016;13(1):49.

62. Spivak AM, Planelles V. HIV-1 Eradication: Early Trials (and Tribulations). Trends Mol Med. 2016;22(1):10-27.

63. Rasmussen TA, Lewin SR. Shocking HIV out of hiding: where are we with clinical trials of latency reversing agents? Curr Opin HIV AIDS. 2016;11(4):394-401.

64. Dental C, Proust A, Ouellet M, Barat C, Tremblay MJ. HIV-1 Latency-Reversing Agents Prostratin and Bryostatin-1 Induce Blood-Brain Barrier Disruption/Inflammation and Modulate Leukocyte Adhesion/Transmigration. J Immunol. 2017;198(3):1229-1241

65. Gama L, et al. Reactivation of simian immunodeficiency virus reservoirs in the brain of virally suppressed macaques. AIDS 2017;31(1):5-14.

66. Jones RB, et al. Histone deacetylase inhibitors impair the elimination of HIV-infected cells by cytotoxic T-lymphocytes. PLoS Pathog. 2014;10(8):e1004287.

67. Farber DL, Yudanin NA, Restifo NP. Human memory T cells: generation, compartmentalization and homeostasis. Nat Rev Immunol. 2014;14(1):24-35.

68. Lee CH, Saksela K, Mirza UA, Chait BT, Kuriyan J. Crystal structure of the conserved core of HIV-1 Nef complexed with a Src family SH3 domain. Cell. 1996;85(6):931-942. 